\title{
Tamed EM schemes for neutral stochastic differential delay equations with superlinear diffusion coefficients
}

\author{
Shounian Deng ${ }^{\mathrm{a}, \mathrm{b}}$, Chen Fei ${ }^{\mathrm{c}}$, Weiyin Fei ${ }^{\mathrm{a}, *}$, Xuerong Mao ${ }^{\mathrm{d}}$ \\ ${ }^{a}$ The Key Laboratory of Advanced Perception and Intelligent Control of High-end Equipment, Ministry of Education, \\ and School of Mathematics and Physics, Anhui Polytechnic University, Wuhu, 241000, China. \\ ${ }^{b}$ School of Science, Nanjing University of Science and Technology, Nanjing, Jiangsu 210094, China \\ ${ }^{c}$ Glorious Sun School of Business and Management, Donghua University, Shanghai, 200051, China \\ ${ }^{d}$ Department of Mathematics and Statistics, University of Strathclyde, Glasgow G1 1XH, UK
}

\begin{abstract}
In this article, we propose two types of explicit tamed Euler-Maruyama (EM) schemes for neutral stochastic differential delay equations with superlinearly growing drift and diffusion coefficients. The first type is convergent in the $\mathcal{L}^{q}$ sense under the local Lipschitz plus Khasminskii-type conditions. The second type is of order half in the mean-square sense under the Khasminskiitype, global monotonicity and polynomial growth conditions. Moreover, it is proved that the partially tamed EM scheme has the property of mean-square exponential stability. Numerical examples are provided to illustrate the theoretical findings.
\end{abstract}

Keywords: Neutral stochastic differential delay equations, tamed EM scheme, super-linear growth, strong convergence, mean-square stability.

\section{Introduction}

As an important type of stochastic differential equations (SDEs), neutral stochastic differential delay equations (NSDDEs) play a significant part in many application fields, such as automatic control, biology, power system and finance [1, 2]. In general, such SDEs with the neutral 5 term do not have any explicit solutions, and we must be content ourselves with an approximation via a numerical approach. Due to the simple algebraic structure, easy implementation and acceptable convergence rate, Euler-type schemes have been introduced to approximate the exact solutions of NSDDEs. Li and Cao [3] presented a two-step Euler-Maruyama (EM) scheme for NSDDEs and studied the mean-square stability of the scheme under the linear growth condition.

10 Mo et al. [4] proposed a split-step theta-method for NSDDEs with Poisson jumps, they also discussed the exponential stability of the method. Ji and Yuan [5] analyzed the convergence rate of tamed EM for NSDDEs with diffusion coefficients of linear growth. Influenced by the work of Mao [6], Lan and Xia [7] developed a modified truncated EM method for SDEs, they extended this method to the case of NSDDEs and obtained the exponential stability of the scheme in [8].

\footnotetext{
${ }^{*}$ Corresponding author

Email address: wyfei@ahpu.edu.cn (Weiyin Fei )
} 
15 Other related work on this topic can be found in $[9,10,11,12,13,14,15,16,17,18]$, and the references therein.

So far as we know, most of the convergence rate results on the numerical schemes for NSDDEs require the conditions that the drift coefficients satisfy the linear or one-sided linear growth condition and the diffusion coefficients satisfy the linear growth condition, see e.g.,

20 [5, 10, 11, 19]. There are limited results on the convergence rate under weaker conditions than these. Zhou and Jin [9] discussed the strong convergence of the backward EM scheme for highly nonlinear NSDDEs, where the diffusion coefficient is polynomially growing with respect to delay term while for non-delay part the coefficient is linearly growing. As a result, our effort is devoted to investigating the strong convergence of explicit numerical approximations, whose

25 convergence order can arrive at one half, for NSDDEs with superlinearly growing drift and diffusion coefficients. It is known that tamed (balanced) or truncation techniques can be used to cope with the superlinearly growing parts appearing in the drift and diffusion coefficients when SDEs are considered. For strong schemes for such SDEs, several types of methods have been introduced: tamed EM schemes, originally proposed by Hutzenthaler et al. in [20], where the

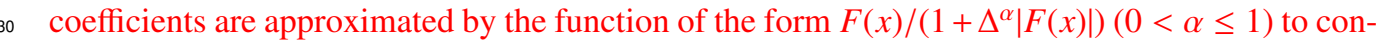
trol their superlinear growth, see e.g., [21, 22, 23, 24, 24, 25, 26, 27, 28]; truncated EM schemes, originally proposed by Mao in [6], where coefficients of superlinear growth can be bounded by the truncated function, see e.g., [29, 30, 31, 32, 33, 34, 35]. Moreover, another method, called Semi-Discrete (SD) method, originally proposed by Halidias [36], also attracts researchers' at35 tention. A major advantage of the SD method is the domain preservation of the solution process, a property that the EM method in general do not preserve [37. There is an ongoing research of the method and its properties, see for instance [38] for an application in a delay model with jumps, and the recent [39] for the convergence order.

Inspired by the taming idea in Sabanis [21] together with the truncation techniques from

40 Mao [29], we propose a class of tamed EM scheme for NSDDEs with coefficients of superlinear growth. According to the scheme we derive some crucial properties P1-P3, which mean that the modified coefficients $f_{\Delta}$ and $g_{\Delta}$ conserve the Khasminskii-type condition and behave linearly for a fixed step size, see (3.11), (3.12) and (3.13). Based on these properties, a uniform moment bound for the numerical solutions is established and then the tamed EM method can be shown to converge strongly and conserve the stability in the mean-square sense.

The main contribution of this paper is to develop two types of explicit tamed EM schemes for NSDDEs, in which both drift and diffusion coefficients can be growing superlinearly, and investigate the strong convergence, mean-square stability of the schemes for NSDDE (2.1). We extend the tamed EM scheme presented in Sabanis [21] to the case of NSDDEs. Furthermore,

50 when the neutral term $D$ is absent in NSDDE (2.1), compared with the convergence results of the truncated EM schemes for stochastic delay differential equations (SDDEs) from Fei et al. [32] Theorem 3.6], our results obtain a better convergence order under almost the same conditions, see Theorem 4.1 .

The rest of the paper is organized as follows. In the next section, we present some preliminaries and assumptions on the NSDDEs. The tamed EM scheme is proposed in Section 3. The discussion of the strong convergence of the tamed EM is given in Section 4. In Section 5, we show the reproduction of mean-square stability of numerical solutions for the exact solution. Numerical examples are presented in Section 6. In the final section, we close the paper by our conclusion. 


\section{Preliminaries}

Throughout this paper, let $(\Omega, \mathcal{F}, \mathbb{P})$ be a complete probability space with a filtration $\left\{\mathcal{F}_{t}\right\}_{t \geq 0}$ satisfying the usual conditions (i.e., it is increasing and right continuous while $\mathcal{F}_{0}$ contains all $\mathbb{P}$-null sets). Let $\tau>0$ be a constant and denote by $C\left([-\tau, 0] ; \mathbb{R}^{d}\right)$ the space of all continuous functions from $[-\tau, 0]$ to $\mathbb{R}^{d}$ with the norm $\|\phi\|=\sup _{-\tau \leq \theta \leq 0}|\phi(\theta)|$. Let $B(t)$ be an $m$-dimensional

65 Brownian motion. If $A$ is a vector or matrix, its transpose is denoted by $A^{T}$. If $X \in \mathbb{R}^{d}$, then $|X|$ is the Euclidean norm. If $A$ is a matrix, its trace norm is denoted by $|A|=\sqrt{\left(A^{T} A\right)}$. For two real numbers $a$ and $b, a \vee b:=\max (a, b)$ and $a \wedge b:=\min (a, b)$. For a set $G$, its indicator function is denoted by $\mathbb{I}_{G}$. The scalar product of two vectors $X, Y \in \mathbb{R}^{d}$ is denoted by $\langle X, Y\rangle$ or $X^{T} Y$. Denote by $\lfloor a\rfloor$ the largest integer which is less or equal to $a$.

Consider a neutral stochastic differential delay equation of the form

$$
d[x(t)-D(x(t-\tau))]=f(x(t)), x(t-\tau)) d t+g(x(t), x(t-\tau)) d B(t), t \geq 0,
$$

with the initial data $\{x(\theta):-\tau \leq \theta \leq 0\}=\xi \in C\left([-\tau, 0] ; \mathbb{R}^{d}\right)$, where $D: \mathbb{R}^{d} \rightarrow \mathbb{R}^{d}, f: \mathbb{R}^{d} \times \mathbb{R}^{d} \rightarrow$ $\mathbb{R}^{d}$ and $g: \mathbb{R}^{d} \times \mathbb{R}^{d} \rightarrow \mathbb{R}^{d \times n}$ are Borel-measurable functions. Unless specified otherwise, we assume that the initial data $\xi$ satisfies the following condition: there is a pair of constants $K_{1}>0$ and $\varrho \in(0,1]$ such that

$$
|\xi(t)-\xi(s)| \leq K_{1}|t-s|^{\varrho}, \forall s, t \in[-\tau, 0] .
$$

Moreover, we assume that $D(0)=0$ and there exists a constant $v \in(0,1)$ such that

$$
|D(x)-D(y)| \leq v|x-y|, \forall x, y \in \mathbb{R}^{d} .
$$

70 Consider the following assumptions:

Assumption 2.1. (Local Lipschitz condition) For any $R>0$, there exists a constant $L_{R}$ depending on $R$ such that

$$
|f(x, y)-f(\bar{x}, \bar{y})| \vee|g(x, y)-g(\bar{x}, \bar{y})| \leq L_{R}(|x-\bar{x}|+|y-\bar{y}|),
$$

$\forall x, \bar{x}, y, \bar{y} \in \mathbb{R}^{d}$ with $|x| \vee|\bar{x}| \vee|y| \vee|\bar{y}| \leq R$.

Assumption 2.2. (Khasminskii-type condition) There exist positive constants $K_{2}$ and $p_{0}>2$ such that

$$
(x-D(y))^{T} f(x, y)+\frac{p_{0}-1}{2}|g(x, y)|^{2} \leq K_{2}\left(1+|x|^{2}+|y|^{2}\right), \quad \forall x, y \in \mathbb{R}^{d} .
$$

Under Assumptions 2.1 and 2.2. NSDDE 2.1) has a unique global solution $x(t)$ on $t \in[-\tau, \infty)$. In addition, we have the following result regarding the moments of $x(t)$, the proof is similar to that of Mao [40, p.213, Theorem 4.5] and is therefore omitted.

Lemma 2.3. Suppose that Assumption 2.1 and 2.2 hold. Then

$$
\sup _{-\tau \leq t \leq T} \mathbb{E}|x(t)|^{p_{0}}<\infty, \forall T>0 .
$$


Denote by $\mathcal{U}$ the family of continuous function $U: \mathbb{R}^{d} \times \mathbb{R}^{d} \rightarrow \mathbb{R}_{+}$such that for any $R>0$, there exists a positive constant $\bar{L}_{R}$ for which

$$
U(x, \bar{x}) \leq \bar{L}_{R}|x-\bar{x}|^{2}, \forall x, \bar{x} \in \mathbb{R}^{d} \text { with }|x| \vee|\bar{x}| \leq R .
$$

Assumption 2.4. (Global monotonicity with $U$ function and polynomial growth conditions) There exist constants $p_{1}>2, l \geq 0$ and $K_{3}>0, K_{4}>0$ as well as a function $U \in \mathcal{U}$ such that

$$
\begin{aligned}
& (x-\bar{x}-D(y)+D(\bar{y}))^{T}(f(x, y)-f(\bar{x}, \bar{y}))+\frac{p_{1}-1}{2}|g(x, y)-g(\bar{x}, \bar{y})|^{2} \\
& \leq K_{3}\left(|x-\bar{x}|^{2}+|y-\bar{y}|^{2}\right)-U(x, \bar{x})+U(y, \bar{y}), \forall x, y \in \mathbb{R}^{d},
\end{aligned}
$$

and

$$
|f(x, y)-f(\bar{x}, \bar{y})| \leq K_{4}\left(1+|x|^{l}+|y|^{l}+|\bar{x}|^{l}+|\bar{y}|^{l}\right)(|x-\bar{x}|+|y-\bar{y}|), \quad \forall x, y \in \mathbb{R}^{d},
$$

as well as

$$
|g(x, y)-g(\bar{x}, \bar{y})|^{2} \leq K_{4}\left(1+|x|^{l}+|y|^{l}+|\bar{x}|^{l}+|\bar{y}|^{l}\right)\left(|x-\bar{x}|^{2}+|y-\bar{y}|^{2}\right), \quad \forall x, y \in \mathbb{R}^{d} .
$$

From 2.6) and 2.7), we have the following growth condition

$$
|f(x, y)| \leq K_{5}\left(1+|x|^{l+1}+|y|^{l+1}\right) \quad \text { and }|g(x, y)|^{2} \leq K_{5}\left(1+|x|^{l+2}+|y|^{l+2}\right), \forall x, y \in \mathbb{R}^{d},
$$

where $K_{5}=6 K_{4} \vee\left(4 K_{4}+2|g(0,0)|^{2}+|f(0,0)|^{2}\right)$.

Remark 2.5. If the neutral term $D$ vanishes, the global monotonicity condition with $U$ function 2.5) reduces to Fei et al. [32] Assumption 2.3] and Guo et al. [34. Assumption 5.1]. In view of Fei et al. [32. Example 6.2], the presence of $U$ function in global monotonicity condition will make the choice of the drift and diffusion coefficients for SDDEs more flexible.

\section{3. Tamed EM scheme for NSDDEs}

Assume that the step size $\Delta$ is a fraction of $\tau$. Define $\Delta=\tau / m \in(0,1]$ for some positive integer $m$ and $\kappa(t):=\lfloor t / \Delta\rfloor \Delta$, for any $t \geq-\tau$. The discrete-time tamed EM scheme for NSDDE (2.1) is defined as follows:

$$
\begin{aligned}
& y_{\Delta}^{k+1}=D\left(y_{\Delta}^{k+1-m}\right)+y_{\Delta}^{k}-D\left(y_{\Delta}^{k-m}\right)+f_{\Delta}\left(y_{\Delta}^{k}, y_{\Delta}^{k-m}\right) \Delta+g_{\Delta}\left(y_{\Delta}^{k}, y_{\Delta}^{k-m}\right) \Delta B_{k}, k=0,1,2, \cdots, \\
& y_{\Delta}^{k}=\xi(k \Delta), k=-m,-m+1, \cdots, 0,
\end{aligned}
$$

where $\Delta B_{k}=B((k+1) \Delta-k \Delta)$, the modified coefficients $f_{\Delta}: \mathbb{R}^{d} \times \mathbb{R}^{d} \rightarrow \mathbb{R}^{d}, g_{\Delta}: \mathbb{R}^{d} \times \mathbb{R}^{d} \rightarrow \mathbb{R}^{d \times n}$ are Borel-measurable and satisfy some conditions given below. Define a continuous-time step process $\bar{Y}_{\Delta}(t)$ on $t \in[-\tau, \infty)$ by

$$
\bar{Y}_{\Delta}(t)=\sum_{k=-m}^{\infty} y_{\Delta}^{k} \mathbb{I}_{[k \Delta,(k+1) \Delta)}(t),
$$


where $\mathbb{I}$ is the indicator function. Then $\bar{Y}_{\Delta}(t-\tau)=y_{\Delta}^{k-m}$, for any $t \in[k \Delta,(k+1) \Delta)$ with $k \geq 0$. Define a new continuous-time process $Y_{\Delta}(t)$ on $t \in[-\tau, \infty)$ by

$$
\begin{aligned}
Y_{\Delta}(t)= & D\left(Y_{\Delta}(t-\tau)\right)+\xi(0)-D(\xi(-\tau))+\int_{0}^{t} f_{\Delta}\left(\bar{Y}_{\Delta}(s), \bar{Y}_{\Delta}(s-\tau)\right) d s \\
& +\int_{0}^{t} g_{\Delta}\left(\bar{Y}_{\Delta}(s), \bar{Y}_{\Delta}(s-\tau)\right) d B(s), t \geq 0, \\
Y_{\Delta}(t)= & \xi(t),-\tau \leq t \leq 0 .
\end{aligned}
$$

Obviously, $Y_{\Delta}(t)$ is well defined on $[0, \tau]$. Once the process $Y_{\Delta}(t)$ on this interval is known, we can proceed this argument on $[\tau, 2 \tau],[2 \tau, 3 \tau]$ etc. and hence obtain the process $Y_{\Delta}(t)$ on the entire interval $[-\tau, \infty)$. Moreover, $Y_{\Delta}(t)$ is an Itô process on $[0, \infty)$ with Itô differential

$$
d\left[Y_{\Delta}(t)-D\left(Y_{\Delta}(t-\tau)\right)\right]=f_{\Delta}\left(\bar{Y}_{\Delta}(t), \bar{Y}_{\Delta}(t-\tau)\right) d t+g_{\Delta}\left(\bar{Y}_{\Delta}(t), \bar{Y}_{\Delta}(t-\tau)\right) d B(t) .
$$

From 3.17 and 3.2), we conclude that

$$
Y_{\Delta}(\Delta)=D(\xi(\Delta-\tau))+\xi(0)-D(\xi(-\tau))+f_{\Delta}(\xi(0), \xi(-\tau)) \Delta+g_{\Delta}(\xi(0), \xi(-\tau)) \Delta B_{0}=y_{\Delta}^{1} .
$$

Similarly, we can show that for any $t \in[k \Delta,(k+1) \Delta)$ with $k \geq 0$,

$$
Y_{\Delta}(k \Delta)=y_{\Delta}^{k}=\bar{Y}_{\Delta}(t),
$$

that is, the discrete and continuous tamed EM solutions coincide at the grid points. Thus, it is useful to know that for any $t \in[k \Delta,(k+1) \Delta)$ with $k \geq 0$,

$$
Y_{\Delta}(k \Delta)-D\left(Y_{\Delta}(k \Delta-\tau)\right)=\bar{Y}_{\Delta}(t)-D\left(\bar{Y}_{\Delta}(t-\tau)\right)=y_{\Delta}^{k}-D\left(y_{\Delta}^{k-m}\right)
$$

and

$$
\begin{aligned}
& Y_{\Delta}(t)-D\left(Y_{\Delta}(t-\tau)\right)-\bar{Y}_{\Delta}(t)+D\left(\bar{Y}_{\Delta}(t-\tau)\right) \\
& =\int_{k \Delta}^{t} f_{\Delta}\left(\bar{Y}_{\Delta}(s), \bar{Y}_{\Delta}(s-\tau)\right) d s+\int_{k \Delta}^{t} g_{\Delta}\left(\bar{Y}_{\Delta}(s), \bar{Y}_{\Delta}(s-\tau)\right) d B(s) .
\end{aligned}
$$

Remark 3.1. In most of the existing work on the numerical methods for NSDDEs, e.g., Ji and Yuan [5], Lan [8], Tan and Yuan [19], $D\left(\bar{Y}_{\Delta}(t-\tau)\right)$, rather than $D\left(Y_{\Delta}(t-\tau)\right)$, as an approximation to $D(x(t-\tau))$, appears in the equation (3.2), which determines another form of continuous-time process $Y_{\Delta}^{*}(t)$ defined by

$$
\begin{aligned}
Y_{\Delta}^{*}(t)= & D\left(\bar{Y}_{\Delta}(t-\tau)\right)+\xi(0)-D(\xi(-\tau))+\int_{0}^{t} f_{\Delta}\left(\bar{Y}_{\Delta}(s), \bar{Y}_{\Delta}(s-\tau)\right) d s \\
& +\int_{0}^{t} g_{\Delta}\left(\bar{Y}_{\Delta}(s), \bar{Y}_{\Delta}(s-\tau)\right) d B(s), t \geq 0 .
\end{aligned}
$$

We then have the following form of difference between the exact solution $x(t)$ and the tamed EM solution $Y_{\Delta}^{*}(t)$,

$$
\begin{aligned}
& x(t)-Y_{\Delta}^{*}(t)-D(x(t-\tau))+D\left(\bar{Y}_{\Delta}(t-\tau)\right) \\
& =\int_{0}^{t}\left(f(x(s), x(s-\tau))-f_{\Delta}\left(\bar{Y}_{\Delta}(s), \bar{Y}_{\Delta}(s-\tau)\right)\right) d s \\
& \quad+\int_{0}^{t}\left(g(x(s), x(s-\tau))-g_{\Delta}\left(\bar{Y}_{\Delta}(s), \bar{Y}_{\Delta}(s-\tau)\right)\right) d B(s), t \geq 0 .
\end{aligned}
$$


If we apply the Itô formula to 3.7 and use the global monotonicity condition with $U$ function (2.5), then there will be an extra term expressed by the following we have to address,

$$
\mathbb{E} \int_{0}^{t}\left(-U\left(x(s), Y_{\Delta}^{*}(s)\right)+U\left(x(s-\tau), Y_{\Delta}^{*}(\kappa(s)-\tau)\right)\right) d s, t \geq 0 .
$$

Note that the two time variables of the second $U$ function in (3.8) are asynchronous, it is difficult to treat (3.8) as an appropriate form. However, if we use $Y_{\Delta}(t)$ given by (3.2), 3.8) becomes

$$
\mathbb{E} \int_{0}^{t}\left(-U\left(x(s), Y_{\Delta}(s)\right)+U\left(x(s-\tau), Y_{\Delta}(s-\tau)\right)\right) d s, t \geq 0 .
$$

We observe from (4.35) that (3.9) can be addressed well. From the practical point of view, using $D\left(Y_{\Delta}(t-\tau)\right)$ to approximate $D(x(t-\tau))$ in (3.7) avoids the presence of asynchronous time. Moreover, $Y_{\Delta}(t)$ preserves the useful property 3.4t that $Y_{\Delta}^{*}(t)$ has.

Let us make some conditions on the coefficients of the scheme 3.1. Suppose that there is a constant $\alpha \in(0,1 / 2]$ such that the following conditions hold:

P1. For any $R>0$, there is a positive constant $N_{R}$ depending on $R$ such that for any $x, y \in \mathbb{R}^{d}$,

$$
\sup _{|x| \vee|y| \leq R}\left|f(x, y)-f_{\Delta}(x, y)\right| \vee\left|g(x, y)-g_{\Delta}(x, y)\right| \leq N_{R} \Delta^{\alpha} .
$$

P2. There is a positive constant $\hat{K}_{1}$ such that for any $\Delta \in(0,1]$,

$$
\left|f_{\Delta}(x, y)\right| \leq \hat{K}_{1} \Delta^{-\alpha}(1+|x|+|y|) \wedge|f(x, y)|, \forall x, y \in \mathbb{R}^{d},
$$

and

$$
\left|g_{\Delta}(x, y)\right|^{2} \leq \hat{K}_{1} \Delta^{-\alpha}\left(1+|x|^{2}+|y|^{2}\right) \wedge|g(x, y)|^{2}, \forall x, y \in \mathbb{R}^{d} .
$$

P3. There is a positive constant $\hat{K}_{2}$ such that

$$
(x-D(y))^{T} f_{\Delta}(x, y)+\frac{p_{0}-1}{2}\left|g_{\Delta}(x, y)\right|^{2} \leq \hat{K}_{2}\left(1+|x|^{2}+|y|^{2}\right), \quad \forall x, y \in \mathbb{R}^{d} .
$$

Property P3 means that modified coefficients $f_{\Delta}$ and $g_{\Delta}$ preserve the Khasminskii-type condition 852.2 While property $\mathrm{P} 2$ implies that for any $\Delta \in(0,1], f_{\Delta}$ and $g_{\Delta}$ satisfy the linear growth condition which guarantees the existence of a unique solution to (3.3).

Now, we propose two types of modified coefficients $f_{\Delta}$ and $g_{\Delta}$ in 3.1$]$. Let $\alpha \in(0,1 / 2]$, define

$$
f_{\Delta}(x, y):=\pi_{\Delta}(x, y) f(x, y) \quad \text { and } \quad g_{\Delta}(x, y):=\pi_{\Delta}(x, y) g(x, y),
$$

where $\pi_{\Delta}: \mathbb{R}^{d} \times \mathbb{R}^{d} \rightarrow(0,1)$ is defined by

$$
\text { Type I: } \quad \pi_{\Delta}(x, y)=\frac{1}{1+\Delta^{\alpha}\left(|f(x, y)|+|g(x, y)|^{2}\right)}, \forall x, y \in \mathbb{R}^{d}, \Delta \in(0,1],
$$

or

$$
\text { Type II: } \quad \pi_{\Delta}(x, y)=\frac{1}{1+\Delta^{\alpha}\left(|x|^{l}+|y|^{l}\right)}, \forall x, y \in \mathbb{R}^{d}, \Delta \in(0,1] .
$$


Remark 3.2. Under Assumptions 2.1 and 2.2. we can show that the modified coefficients $f_{\Delta}$ and $g_{\Delta}$ with Type I given by (3.15) satisfy conditions P1-P3. If Assumptions 2.1 is replaced by Assumption 2.4 the modified coefficients Type II also satisfy P1-P3. This type of tamed EM

90 scheme allows us to produce the optimal rate of convergence. But if we are only interested in the strong convergence (without order) of the numerical scheme, then using the tamed EM Type I may suffice.

\section{Strong convergence at time $T>0$}

4.1. Order of strong convergence of tamed EM (Type II) under monotonicity condition

Theorem 4.1. Suppose that Assumptions 2.2 and 2.4 hold with $p_{0} \geq 4 \vee 2(1+2 l)$ and $\alpha \in$ $(0,1 / 2]$ is arbitrary. Then the tamed EM solution $Y_{\Delta}(t)$ or $\bar{Y}_{\Delta}(t)$ with modified coefficients Type II converges to the exact solution $x(t)$ of NSDDE 2.1] with order $\alpha \wedge \varrho$ in the mean-square sense, i.e.,

$$
\sup _{0 \leq t \leq T} \mathbb{E}\left|x(t)-Y_{\Delta}(t)\right|^{2} \leq C \Delta^{2(\alpha \wedge \varrho)} \quad \text { and } \quad \sup _{0 \leq t \leq T} \mathbb{E}\left|x(t)-\bar{Y}_{\Delta}(t)\right|^{2} \leq C \Delta^{2(\alpha \wedge \varrho)}, \forall \Delta \in(0,1],
$$

where the positive constant $C:=C\left(T, v,\|\xi\|, p_{0}, p_{1}, K_{1}, K_{2}, K_{3}, K_{4}, l\right)$. In particular, letting $\alpha=$ $1 / 2$ yields that

$$
\left[\mathbb{E}\left|x(T)-Y_{\Delta}(T)\right|^{2}\right]^{1 / 2} \leq C \Delta^{0.5 \wedge \varrho} \quad \text { and } \quad\left[\mathbb{E}\left|x(T)-\bar{Y}_{\Delta}(T)\right|^{2}\right]^{1 / 2} \leq C \Delta^{0.5 \wedge \varrho}, \forall \Delta \in(0,1] .
$$

95 From now on, $C$ denotes a genetic positive real constant dependent on $T, v,\|\xi\|$ etc. but independent of $\Delta$.

Remark 4.2. It should be pointed out that if the neutral term D vanishes in NSDDE (2.1), Theorem 4.1 reduces to the convergence result of tamed EM scheme for SDDEs. In this case, compared with the convergence result of the truncated EM scheme in Fei et al. [32. Corollary 3.8], which has an order $(0.5-\varepsilon) \wedge \varrho$ in the mean-square sense, where $\varepsilon \in(0,1 / 4]$ is arbitraty, we observe from (4.2) that our scheme has a better convergence order under almost the same conditions as [32] Corollary 3.8].

Lemma 4.3. Suppose that $P 2$ and $P 3$ hold with $p_{0} \geq 4$ and $\alpha \in(0,1 / 2]$ is arbitrary. Then the tamed EM solution $Y_{\Delta}(t)$ defined by (3.2) satisfies

$$
\sup _{0<\Delta \leq 1} \sup _{0 \leq t \leq T} \mathbb{E}\left|Y_{\Delta}(t)\right|^{p_{0}} \leq C,
$$

where the positive constant $C:=C\left(p_{0}, T, K_{2},\|\xi\|, v\right)$. 
Proof. Let P2 and P3 hold with $p_{0} \geq 4$ and $\alpha \in(0,1 / 2]$. Then for any $t \in[0, T]$, applying the Itô formula to 3.2 and P3, i.e., 3.13, we derive that

$$
\begin{aligned}
& \mathbb{E}\left|Y_{\Delta}(t)-D\left(Y_{\Delta}(t-\tau)\right)\right|^{p_{0}} \\
& \leq|\xi(0)-D(\xi(-\tau))|^{p_{0}}+p_{0} \mathbb{E} \int_{0}^{t}\left[\left|Y_{\Delta}(s)-D\left(Y_{\Delta}(s-\tau)\right)\right|^{p_{0}-2}\right. \\
& \left.\times\left(\left[Y_{\Delta}(s)-D\left(Y_{\Delta}(s-\tau)\right)\right]^{T} f_{\Delta}\left(\bar{Y}_{\Delta}(s), \bar{Y}_{\Delta}(s-\tau)\right)+\frac{p_{0}-1}{2}\left|g_{\Delta}\left(\bar{Y}_{\Delta}(s), \bar{Y}_{\Delta}(s-\tau)\right)\right|^{2}\right)\right] d s \\
& =|\xi(0)-D(\xi(-\tau))|^{p_{0}}+p_{0} \mathbb{E} \int_{0}^{t}\left[\left|Y_{\Delta}(s)-D\left(Y_{\Delta}(s-\tau)\right)\right|^{p_{0}-2}\right. \\
& \left.\times\left(\left[\bar{Y}_{\Delta}(s)-D\left(\bar{Y}_{\Delta}(s-\tau)\right)\right]^{T} f_{\Delta}\left(\bar{Y}_{\Delta}(s), \bar{Y}_{\Delta}(s-\tau)\right)+\frac{p_{0}-1}{2}\left|g_{\Delta}\left(\bar{Y}_{\Delta}(s), \bar{Y}_{\Delta}(s-\tau)\right)\right|^{2}\right)\right] d s \\
& +p_{0} \mathbb{E} \int_{0}^{t}\left[\left|Y_{\Delta}(s)-D\left(Y_{\Delta}(s-\tau)\right)\right|^{p_{0}-2}\right. \\
& \left.\times\left[Y_{\Delta}(s)-D\left(Y_{\Delta}(s-\tau)\right)-\bar{Y}_{\Delta}(s)+D\left(\bar{Y}_{\Delta}(s-\tau)\right)\right]^{T} f_{\Delta}\left(\bar{Y}_{\Delta}(s), \bar{Y}_{\Delta}(s-\tau)\right)\right] d s \\
& \leq|\xi(0)-D(\xi(-\tau))|^{p_{0}}+C \mathbb{E} \int_{0}^{t}\left[\left|Y_{\Delta}(s)-D\left(Y_{\Delta}(s-\tau)\right)\right|^{p_{0}-2}\left(1+\left|\bar{Y}_{\Delta}(s)\right|^{2}+\left|\bar{Y}_{\Delta}(s-\tau)\right|^{2}\right)\right] d s+p_{0} J(t) \\
& \leq C+C \mathbb{E} \int_{0}^{t}\left[\left(\left|Y_{\Delta}(s)\right|^{p_{0}-2}+\left|D\left(Y_{\Delta}(s-\tau)\right)\right|^{p_{0}-2}\right)\left(1+\left|\bar{Y}_{\Delta}(s)\right|^{2}+\left|\bar{Y}_{\Delta}(s-\tau)\right|^{2}\right)\right] d s+p_{0} J(t) \\
& \leq C+C \mathbb{E} \int_{0}^{t}\left[1+\left|Y_{\Delta}(s)\right|^{p_{0}}+\left|Y_{\Delta}(s-\tau)\right|^{p_{0}}+\left|\bar{Y}_{\Delta}(s)\right|^{p_{0}}+\left|\bar{Y}_{\Delta}(s-\tau)\right|^{p_{0}}\right] d s+p_{0} J(t) \\
& \leq C+C \int_{0}^{t}\left[\|\xi\|^{p_{0}}+\sup _{0 \leq u \leq s} \mathbb{E}\left|Y_{\Delta}(u)\right|^{p_{0}}+\sup _{0 \leq u \leq s} \mathbb{E}\left|\bar{Y}_{\Delta}(u)\right|^{p_{0}}\right] d s+p_{0} J(t),
\end{aligned}
$$

where

$$
\begin{aligned}
J(t):= & \mathbb{E} \int_{0}^{t}\left[\left|Y_{\Delta}(s)-D\left(Y_{\Delta}(s-\tau)\right)\right|^{p_{0}-2}\right. \\
& \left.\times\left(Y_{\Delta}(s)-D\left(Y_{\Delta}(s-\tau)\right)-\bar{Y}_{\Delta}(s)+D\left(\bar{Y}_{\Delta}(s-\tau)\right)\right)^{T} f_{\Delta}\left(\bar{Y}_{\Delta}(s), \bar{Y}_{\Delta}(s-\tau)\right)\right] d s .
\end{aligned}
$$

From 3.4, we have the following useful estimate:

$$
\sup _{0 \leq u \leq s} \mathbb{E}\left|\bar{Y}_{\Delta}(u)\right|^{p_{0}} \leq \sup _{0 \leq u \leq s} \mathbb{E}\left|Y_{\Delta}(u)\right|^{p_{0}}
$$

Consequently,

$$
\mathbb{E}\left|Y_{\Delta}(t)-D\left(Y_{\Delta}(t-\tau)\right)\right|^{p_{0}} \leq C+C \int_{0}^{t}\left[\|\xi\|^{p_{0}}+\sup _{0 \leq u \leq s} \mathbb{E}\left|Y_{\Delta}(u)\right|^{p_{0}}\right] d s+p_{0} J(t) .
$$

We observe that

$$
\begin{aligned}
& J(t)= \mathbb{E} \int_{0}^{t}\left[\left|\bar{Y}_{\Delta}(s)-D\left(\bar{Y}_{\Delta}(s-\tau)\right)\right|^{p_{0}-2}\right. \\
&\left.\times\left(Y_{\Delta}(s)-D\left(Y_{\Delta}(s-\tau)\right)-\bar{Y}_{\Delta}(s)+D\left(\bar{Y}_{\Delta}(s-\tau)\right)\right)^{T} f_{\Delta}\left(\bar{Y}_{\Delta}(s), \bar{Y}_{\Delta}(s-\tau)\right)\right] d s \\
&+\mathbb{E} \int_{0}^{t}\left[\left(\left|Y_{\Delta}(s)-D\left(Y_{\Delta}(s-\tau)\right)\right|^{p_{0}-2}-\left|\bar{Y}_{\Delta}(s)-D\left(\bar{Y}_{\Delta}(s-\tau)\right)\right|^{p_{0}-2}\right)\right. \\
& 8
\end{aligned}
$$




$$
\begin{aligned}
& \left.\times\left(Y_{\Delta}(s)-D\left(Y_{\Delta}(s-\tau)\right)-\bar{Y}_{\Delta}(s)+D\left(\bar{Y}_{\Delta}(s-\tau)\right)\right)^{T} f_{\Delta}\left(\bar{Y}_{\Delta}(s), \bar{Y}_{\Delta}(s-\tau)\right)\right] d s \\
= & : J_{1}(t)+J_{2}(t) .
\end{aligned}
$$

Using (3.6) and P2, we have the following estimate

$$
\begin{aligned}
J_{1}(t)= & \mathbb{E} \int_{0}^{t}\left[\left|\bar{Y}_{\Delta}(s)-D\left(\bar{Y}_{\Delta}(s-\tau)\right)\right|^{p_{0}-2}\left(\int_{\kappa(s)}^{s} f_{\Delta}\left(\bar{Y}_{\Delta}(u), \bar{Y}_{\Delta}(u-\tau)\right) d u\right)^{T} f_{\Delta}\left(\bar{Y}_{\Delta}(s), \bar{Y}_{\Delta}(s-\tau)\right)\right] d s \\
& +\mathbb{E} \int_{0}^{t}\left[\left|\bar{Y}_{\Delta}(s)-D\left(\bar{Y}_{\Delta}(s-\tau)\right)\right|^{p_{0}-2}\left(\int_{\kappa(s)}^{s} g_{\Delta}\left(\bar{Y}_{\Delta}(u), \bar{Y}_{\Delta}(u-\tau)\right) d B(u)\right)^{T} f_{\Delta}\left(\bar{Y}_{\Delta}(s), \bar{Y}_{\Delta}(s-\tau)\right)\right] d s \\
\leq & \mathbb{E} \int_{0}^{t}\left[\left|\bar{Y}_{\Delta}(s)-D\left(\bar{Y}_{\Delta}(s-\tau)\right)\right|^{p_{0}-2}\left|f_{\Delta}\left(\bar{Y}_{\Delta}(s), \bar{Y}_{\Delta}(s-\tau)\right)\right|^{2} \int_{\kappa(s)}^{s} d u\right] d s \\
\leq & C \Delta^{1-2 \alpha} \mathbb{E} \int_{0}^{t}\left|\bar{Y}_{\Delta}(s)-D\left(\bar{Y}_{\Delta}(s-\tau)\right)\right|^{p_{0}-2}\left(1+\left|\bar{Y}_{\Delta}(s)\right|^{2}+\left|\bar{Y}_{\Delta}(s-\tau)\right|^{2}\right) d s \\
\leq & C \int_{0}^{t}\left[1+\sup _{0 \leq u \leq s} \mathbb{E}\left|\bar{Y}_{\Delta}(u)-D\left(\bar{Y}_{\Delta}(u-\tau)\right)\right|^{p_{0}}+\sup _{0 \leq u \leq s} \mathbb{E}\left|\bar{Y}_{\Delta}(u)\right|^{p_{0}}+\sup _{0 \leq u \leq s} \mathbb{E}\left|\bar{Y}_{\Delta}(u-\tau)\right|^{p_{0}}\right] d s \\
\leq & C \int_{0}^{t}\left[1+\|\xi\|^{p_{0}}+\sup _{0 \leq u \leq s} \mathbb{E}\left|\bar{Y}_{\Delta}(u)-D\left(\bar{Y}_{\Delta}(u-\tau)\right)\right|^{p_{0}}+\sup _{0 \leq u \leq s} \mathbb{E}\left|\bar{Y}_{\Delta}(u)\right|^{p_{0}}\right] d s \\
\leq & C+C \int_{0}^{t}\left[\|\xi\|^{p_{0}}+\sup _{0 \leq u \leq s} \mathbb{E}\left|Y_{\Delta}(u)\right|^{p_{0}}\right] d s,
\end{aligned}
$$

where 4.4 has been used in the derivation of the last inequality.

For some $p_{0} \geq 4$, applying the Itô formula to $\left|Y_{\Delta}(s)-D\left(Y_{\Delta}(s-\tau)\right)\right|^{p_{0}-2}$, we conclude from (3.2) or 3.6 that

$$
\begin{aligned}
J_{2}(t)= & \mathbb{E} \int_{0}^{t}\left[\left(\left|Y_{\Delta}(s)-D\left(Y_{\Delta}(s-\tau)\right)\right|^{p_{0}-2}-\left|\bar{Y}_{\Delta}(s)-D\left(\bar{Y}_{\Delta}(s-\tau)\right)\right|^{p_{0}-2}\right)\right. \\
& \left.\times\left(Y_{\Delta}(s)-D\left(Y_{\Delta}(s-\tau)\right)-\bar{Y}_{\Delta}(s)+D\left(\bar{Y}_{\Delta}(s-\tau)\right)\right)^{T} f_{\Delta}\left(\bar{Y}_{\Delta}(s), \bar{Y}_{\Delta}(s-\tau)\right)\right] d s \\
\leq & \mathbb{E} \int_{0}^{t}\left[\left\{( p _ { 0 } - 2 ) \int _ { \kappa ( s ) } ^ { s } | Y _ { \Delta } ( u ) - D ( Y _ { \Delta } ( u - \tau ) ) | ^ { p _ { 0 } - 4 } \left(Y_{\Delta}(u)-D\left(Y_{\Delta}(u-\tau)\right)^{T} f_{\Delta}\left(\bar{Y}_{\Delta}(u), \bar{Y}_{\Delta}(u-\tau)\right) d u\right.\right.\right. \\
& +\frac{\left(p_{0}-2\right)\left(p_{0}-3\right)}{2} \int_{\kappa(s)}^{s}\left|Y_{\Delta}(u)-D\left(Y_{\Delta}(u-\tau)\right)\right|^{p_{0}-4}\left|g_{\Delta}\left(\bar{Y}_{\Delta}(u), \bar{Y}_{\Delta}(u-\tau)\right)\right|^{2} d u \\
& +\left(p_{0}-2\right) \int_{\kappa(s)}^{s}\left|Y_{\Delta}(u)-D\left(Y_{\Delta}(u-\tau)\right)\right|^{p_{0}-4}\left(Y_{\Delta}(u)-D\left(Y_{\Delta}(u-\tau)\right)^{T} g_{\Delta}\left(\bar{Y}_{\Delta}(u), \bar{Y}_{\Delta}(u-\tau)\right) d B(u)\right\} \\
& \left.\times\left(\int_{\kappa(s)}^{s} f_{\Delta}\left(\bar{Y}_{\Delta}(u), \bar{Y}_{\Delta}(u-\tau)\right) d u+\int_{\kappa(s)}^{s} g_{\Delta}\left(\bar{Y}_{\Delta}(u), \bar{Y}_{\Delta}(u-\tau)\right) d B(u)\right)^{T} f_{\Delta}\left(\bar{Y}_{\Delta}(s), \bar{Y}_{\Delta}(s-\tau)\right)\right] d s \\
= & \sum_{i=1}^{6} J_{2 i}(t),
\end{aligned}
$$

where

$J_{21}(t)=C \mathbb{E} \int_{0}^{t}\left[\int_{\kappa(s)}^{s}\left|Y_{\Delta}(u)-D\left(Y_{\Delta}(u-\tau)\right)\right|^{p_{0}-4}\left(Y_{\Delta}(u)-D\left(Y_{\Delta}(u-\tau)\right)^{T} f_{\Delta}\left(\bar{Y}_{\Delta}(u), \bar{Y}_{\Delta}(u-\tau)\right) d u\right.\right.$ 


$$
\begin{aligned}
& \left.\times\left(\int_{\kappa(s)}^{s} f_{\Delta}\left(\bar{Y}_{\Delta}(s), \bar{Y}_{\Delta}(s-\tau)\right) d u\right)^{T} f_{\Delta}\left(\bar{Y}_{\Delta}(s), \bar{Y}_{\Delta}(s-\tau)\right)\right] d s \\
J_{22}(t)= & C \mathbb{E} \int_{0}^{t}\left[\int _ { \kappa ( s ) } ^ { s } | Y _ { \Delta } ( u ) - D ( Y _ { \Delta } ( u - \tau ) ) | ^ { p _ { 0 } - 4 } \left(Y_{\Delta}(u)-D\left(Y_{\Delta}(u-\tau)\right)^{T} f_{\Delta}\left(\bar{Y}_{\Delta}(u), \bar{Y}_{\Delta}(u-\tau)\right) d u\right.\right. \\
& \left.\times\left(\int_{\kappa(s)}^{s} g_{\Delta}\left(\bar{Y}_{\Delta}(s), \bar{Y}_{\Delta}(s-\tau)\right) d B(u)\right)^{T} f_{\Delta}\left(\bar{Y}_{\Delta}(s), \bar{Y}_{\Delta}(s-\tau)\right)\right] d s \\
J_{23}(t)= & C \mathbb{E} \int_{0}^{t}\left[\int_{\kappa(s)}^{s}\left|Y_{\Delta}(u)-D\left(Y_{\Delta}(u-\tau)\right)\right|^{p_{0}-4}\left|g_{\Delta}\left(\bar{Y}_{\Delta}(u), \bar{Y}_{\Delta}(u-\tau)\right)\right|^{2} d u\right. \\
& \left.\times\left(\int_{\kappa(s)}^{s} f_{\Delta}\left(\bar{Y}_{\Delta}(s), \bar{Y}_{\Delta}(s-\tau)\right) d u\right)^{T} f_{\Delta}\left(\bar{Y}_{\Delta}(s), \bar{Y}_{\Delta}(s-\tau)\right)\right] d s \\
J_{24}(t)= & C \mathbb{E} \int_{0}^{t}\left[\int_{\kappa(s)}^{s}\left|Y_{\Delta}(u)-D\left(Y_{\Delta}(u-\tau)\right)\right|^{p_{0}-4}\left|g_{\Delta}\left(\bar{Y}_{\Delta}(u), \bar{Y}_{\Delta}(u-\tau)\right)\right|^{2} d u\right. \\
& \left.\times\left(\int_{\kappa(s)}^{s} g_{\Delta}\left(\bar{Y}_{\Delta}(u), \bar{Y}_{\Delta}(u-\tau)\right) d B(u)\right)^{T} f_{\Delta}\left(\bar{Y}_{\Delta}(s), \bar{Y}_{\Delta}(s-\tau)\right)\right] d s \\
J_{25}(t)= & C \mathbb{E} \int_{0}^{t}\left[\int _ { \kappa ( s ) } ^ { s } | Y _ { \Delta } ( u ) - D ( Y _ { \Delta } ( u - \tau ) ) | ^ { p _ { 0 } - 4 } \left(Y_{\Delta}(u)-D\left(Y_{\Delta}(u-\tau)\right)^{T} g_{\Delta}\left(\bar{Y}_{\Delta}(u), \bar{Y}_{\Delta}(u-\tau)\right) d B(u)\right.\right. \\
& \left.\times\left(\int_{\kappa(s)}^{s} f_{\Delta}\left(\bar{Y}_{\Delta}(u), \bar{Y}_{\Delta}(u-\tau)\right) d u\right)^{T} f_{\Delta}\left(\bar{Y}_{\Delta}(s), \bar{Y}_{\Delta}(s-\tau)\right)\right] d s \\
J_{26}(t)= & C \mathbb{E} \int_{0}^{t}\left[\int _ { \kappa ( s ) } ^ { s } | Y _ { \Delta } ( u ) - D ( Y _ { \Delta } ( u - \tau ) ) | ^ { p _ { 0 } - 4 } \left(Y_{\Delta}(u)-D\left(Y_{\Delta}(u-\tau)\right)^{T} g_{\Delta}\left(\bar{Y}_{\Delta}(u), \bar{Y}_{\Delta}(u-\tau)\right) d B(u)\right.\right. \\
& \left.\times\left(\int_{\kappa(s)}^{s} g_{\Delta}\left(\bar{Y}_{\Delta}(u), \bar{Y}_{\Delta}(u-\tau)\right) d B(u)\right)^{T} f_{\Delta}\left(\bar{Y}_{\Delta}(s), \bar{Y}_{\Delta}(s-\tau)\right)\right] d s .
\end{aligned}
$$

By P2 and 4.4), we have

$$
\begin{aligned}
J_{21}(t) & \leq C \mathbb{E} \int_{0}^{t}\left[\left|f_{\Delta}\left(\bar{Y}_{\Delta}(s), \bar{Y}_{\Delta}(s-\tau)\right)\right|^{2} \Delta \int_{\kappa(s)}^{s}\left|Y_{\Delta}(u)-D\left(Y_{\Delta}(u-\tau)\right)\right|^{p_{0}-3}\left|f_{\Delta}\left(\bar{Y}_{\Delta}(u), \bar{Y}_{\Delta}(u-\tau)\right)\right| d u\right] d s \\
& \leq C \Delta \mathbb{E} \int_{0}^{t}\left[\left|f_{\Delta}\left(\bar{Y}_{\Delta}(s), \bar{Y}_{\Delta}(s-\tau)\right)\right|^{3} \int_{\kappa(s)}^{s}\left|Y_{\Delta}(u)-D\left(Y_{\Delta}(u-\tau)\right)\right|^{p_{0}-3} d u\right] d s \\
& \leq C \Delta^{1-3 \alpha} \mathbb{E} \int_{0}^{t}\left[\int_{\kappa(s)}^{s}\left(1+|\bar{Y}(\kappa(s))|^{3}+|\bar{Y}(\kappa(s)-\tau)|^{3}\right)\left|Y_{\Delta}(u)-D\left(Y_{\Delta}(u-\tau)\right)\right|^{p_{0}-3} d u\right] d s \\
& \leq C \Delta^{2-3 \alpha} \int_{0}^{t}\left[1+\sup _{0 \leq u \leq s} \mathbb{E}\left|Y_{\Delta}(u)-D\left(Y_{\Delta}(u-\tau)\right)\right|^{p_{0}}+\sup _{0 \leq u \leq s} \mathbb{E}\left|\bar{Y}_{\Delta}(u)\right|^{p_{0}}+\sup _{0 \leq u \leq s} \mathbb{E}\left|\bar{Y}_{\Delta}(u-\tau)\right|^{p_{0}}\right] d s \\
& \leq C+C \int_{0}^{t}\left[\|\xi\|^{p_{0}}+\sup _{0 \leq u \leq s} \mathbb{E}\left|Y_{\Delta}(u)\right|^{p_{0}}\right] d s .
\end{aligned}
$$

Recall the Young inequality: for $r_{1}^{-1}+r_{2}^{-1}=1, r_{1}, r_{2}>1$,

$$
a b \leq \frac{a^{r_{1}}}{r_{1}}+\frac{b^{r_{2}}}{r_{2}}, \forall a, b>0 .
$$


Letting $r_{1}=p_{0}, r_{2}=\frac{p_{0}}{p_{0}-1}$ in the above inequality, applying the Hölder and Burkholder-DavisGundy inequalities, we have

$$
\begin{aligned}
J_{22}(t) \leq & C \mathbb{E} \int_{0}^{t}\left[\int_{\kappa(s)}^{s}\left|Y_{\Delta}(u)-D\left(Y_{\Delta}(u-\tau)\right)\right|^{p_{0}-3}\left|f_{\Delta}\left(\bar{Y}_{\Delta}(u), \bar{Y}_{\Delta}(u-\tau)\right)\right| d u\right. \\
& \left.\times\left|\int_{\kappa(s)}^{s} g_{\Delta}\left(\bar{Y}_{\Delta}(u), \bar{Y}_{\Delta}(u-\tau)\right) d B(u)\right|\left|f_{\Delta}\left(\bar{Y}_{\Delta}(s), \bar{Y}_{\Delta}(s-\tau)\right)\right|\right] d s \\
\leq & C \mathbb{E} \int_{0}^{t}\left[\left(\left|f_{\Delta}\left(\bar{Y}_{\Delta}(s), \bar{Y}_{\Delta}(s-\tau)\right)\right| \int_{\kappa(s)}^{s}\left|Y_{\Delta}(u)-D\left(Y_{\Delta}(u-\tau)\right)\right|^{p_{0}-3}\left|f_{\Delta}\left(\bar{Y}_{\Delta}(u), \bar{Y}_{\Delta}(u-\tau)\right)\right| d u\right)^{p_{0} /\left(p_{0}-1\right)}\right. \\
+ & \left.\left|\int_{\kappa(s)}^{s} g_{\Delta}\left(\bar{Y}_{\Delta}(u), \bar{Y}_{\Delta}(u-\tau)\right) d B(u)\right|^{p_{0}}\right] d s \\
\leq & C \mathbb{E} \int_{0}^{t}\left[\left(\Delta^{-2 \alpha} \int_{\kappa(s)}^{s}\left|Y_{\Delta}(u)-D\left(Y_{\Delta}(u-\tau)\right)\right|^{p_{0}-3}\left(1+\left|\bar{Y}_{\Delta}(s)\right|^{2}+\left|\bar{Y}_{\Delta}(s-\tau)\right|^{2}\right) d u\right)^{p_{0} /\left(p_{0}-1\right)}\right] d s \\
& +C \int_{0}^{t}\left[\mathbb{E}\left(\int_{\kappa(s)}^{s}\left|g_{\Delta}\left(\bar{Y}_{\Delta}(u), \bar{Y}_{\Delta}(u-\tau)\right)\right|^{2} d u\right)^{p_{0} / 2}\right] d s \\
\leq & C \Delta^{(1-2 \alpha) p_{0} /\left(p_{0}-1\right)} \int_{0}^{t}\left[1+\sup _{0 \leq u \leq s} \mathbb{E}\left|Y_{\Delta}(u)-D\left(Y_{\Delta}(u-\tau)\right)\right|^{p_{0}}+\sup _{0 \leq u \leq s} \mathbb{E}\left|\bar{Y}_{\Delta}(u)\right|^{p_{0}}\right. \\
& \left.+\sup _{0 \leq u \leq s} \mathbb{E}\left|\bar{Y}_{\Delta}(u-\tau)\right|^{p_{0}}\right] d s+C \Delta^{(1-\alpha) p_{0} / 2} \int_{0}^{t}\left[1+\sup _{0 \leq u \leq s} \mathbb{E}\left|\bar{Y}_{\Delta}(u)\right|^{p_{0}}+\sup _{0 \leq u \leq s}{\left.\mathbb{E}\left|\bar{Y}_{\Delta}(u-\tau)\right|^{p_{0}}\right] d s}_{\leq} C+C \int_{0}^{t}\left[\|\xi\|^{p_{0}}+\sup _{0 \leq u \leq s} \mathbb{E}\left|Y_{\Delta}(u)\right|^{p_{0}}\right] d s .\right.
\end{aligned}
$$

Again using P2 and noting that $\alpha \in(0,1 / 2]$, we have

$$
\begin{aligned}
J_{26}(t)= & C \mathbb{E} \int_{0}^{t}\left[\int_{\kappa(s)}^{s} \mid Y_{\Delta}(u)-D\left(Y _ { \Delta } ( u - \tau ) | ^ { p _ { 0 } - 4 } \left(Y_{\Delta}(u)-D\left(Y_{\Delta}(u-\tau)\right)^{T}\left|g_{\Delta}\left(\bar{Y}_{\Delta}(u), \bar{Y}_{\Delta}(u-\tau)\right)\right|^{2} d u\right.\right.\right. \\
& \left.\times f_{\Delta}\left(\bar{Y}_{\Delta}(s), \bar{Y}_{\Delta}(s-\tau)\right)\right] d s \\
\leq & \mathbb{E} \int_{0}^{t}\left[\int_{\kappa(s)}^{s} \mid Y_{\Delta}(u)-D\left(\left.Y_{\Delta}(u-\tau)\right|^{p_{0}-3}\left|g_{\Delta}\left(\bar{Y}_{\Delta}(u), \bar{Y}_{\Delta}(u-\tau)\right)\right|^{2} d u\left|f_{\Delta}\left(\bar{Y}_{\Delta}(s), \bar{Y}_{\Delta}(s-\tau)\right)\right|\right] d s\right. \\
\leq & C \Delta^{-2 \alpha} \mathbb{E} \int_{0}^{t}\left[\int_{\kappa(s)}^{s} \mid Y_{\Delta}(u)-D\left(\left.Y_{\Delta}(u-\tau)\right|^{p_{0}-3} \mid\left(1+\left|\bar{Y}_{\Delta}(u)\right|^{2}+|\bar{Y}(u-\tau)|^{2}\right) d u\right.\right. \\
& \left.\times\left(1+\left|\bar{Y}_{\Delta}(s)\right|+|\bar{Y}(s-\tau)|\right)\right] d s \\
\leq & C \Delta^{1-2 \alpha} \int_{0}^{t}\left[1+\sup _{0 \leq u \leq s} \mathbb{E}\left|Y_{\Delta}(u)-D\left(Y_{\Delta}(u-\tau)\right)\right|^{p_{0}}+\sup _{0 \leq u \leq s} \mathbb{E}\left|\bar{Y}_{\Delta}(u)\right|^{p_{0}}+\sup _{0 \leq u \leq s} \mathbb{E}\left|\bar{Y}_{\Delta}(u-\tau)\right|^{p_{0}}\right] d s \\
\leq & C+C \int_{0}^{t}\left[\|\xi\|^{p_{0}}+\sup _{0 \leq u \leq s} \mathbb{E}\left|Y_{\Delta}(u)\right|^{p_{0}}\right] d s .
\end{aligned}
$$

Similarly, we can derive that

$$
J_{23}(t)+J_{24}(t)+J_{25}(t) \leq C+C \int_{0}^{t}\left[\|\xi\|^{p_{0}}+\sup _{0 \leq u \leq s} \mathbb{E}\left|Y_{\Delta}(u)\right|^{p_{0}}\right] d s .
$$


From 4.9-4.12, we have

$$
J_{2}(t) \leq C+C \int_{0}^{t}\left[\|\xi\|^{p_{0}}+\sup _{0 \leq u \leq s} \mathbb{E}\left|Y_{\Delta}(u)\right|^{p_{0}}\right] d s .
$$

Inserting 4.7) and 4.13 into 4.6, we derive from 4.5) that

$$
\sup _{0 \leq u \leq t} \mathbb{E}\left|Y_{\Delta}(u)-D\left(Y_{\Delta}(u-\tau)\right)\right|^{p_{0}} \leq C+C \int_{0}^{t} \sup _{0 \leq u \leq s} \mathbb{E}\left|Y_{\Delta}(u)\right|^{p_{0}} d s .
$$

Recall the following inequality: for $p>1, \varepsilon>0$ and $a, b \in \mathbb{R}$,

$$
|a+b|^{p} \leq\left(1+\varepsilon^{\frac{1}{p-1}}\right)^{p-1}\left(\frac{|a|^{p}}{\varepsilon}+|b|^{p}\right),
$$

see [40, Lemma 4.1, p.211]. Consequently,

$$
\begin{aligned}
\left|Y_{\Delta}(u)\right|^{p_{0}} & =\left|Y_{\Delta}(u)-D\left(Y_{\Delta}(u-\tau)\right)+D\left(Y_{\Delta}(u-\tau)\right)\right|^{p_{0}} \\
& \leq\left(1+\varepsilon^{\frac{1}{p_{0}-1}}\right)^{p_{0}-1}\left(\frac{\left|D\left(Y_{\Delta}(u-\tau)\right)\right|^{p_{0}}}{\varepsilon}+\left|Y_{\Delta}(u)-D\left(Y_{\Delta}(u-\tau)\right)\right|^{p_{0}}\right) \\
& \leq\left(1+\varepsilon^{\frac{1}{p_{0}-1}}\right)^{p_{0}-1}\left(\frac{v^{p_{0}}\left|\left(Y_{\Delta}(u-\tau)\right)\right|^{p_{0}}}{\varepsilon}+\left|Y_{\Delta}(u)-D\left(Y_{\Delta}(u-\tau)\right)\right|^{p_{0}}\right) .
\end{aligned}
$$

Letting $\varepsilon=\left(\frac{v}{1-v}\right)^{p_{0}-1}$ and taking expectations, we have

$$
\mathbb{E}\left|Y_{\Delta}(u)\right|^{p_{0}} \leq v \mathbb{E}\left|Y_{\Delta}(u-\tau)\right|^{p_{0}}+\frac{1}{(1-v)^{p_{0}-1}} \mathbb{E}\left|Y_{\Delta}(u)-D\left(Y_{\Delta}(u-\tau)\right)\right|^{p_{0}}, \quad \forall u \geq 0 .
$$

Therefore,

$$
\begin{aligned}
\sup _{0 \leq u \leq s} \mathbb{E}\left|Y_{\Delta}(u)\right|^{p_{0}} & \leq v \sup _{0 \leq u \leq s} \mathbb{E}\left|Y_{\Delta}(u-\tau)\right|^{p_{0}}+\frac{1}{(1-v)^{p_{0}-1}} \sup _{0 \leq u \leq s} \mathbb{E}\left|Y_{\Delta}(u)-D\left(Y_{\Delta}(u-\tau)\right)\right|^{p_{0}} \\
& \leq v\|\xi\|^{p_{0}}+v \sup _{0 \leq u \leq s} \mathbb{E}\left|Y_{\Delta}(u)\right|^{p_{0}}+\frac{1}{(1-v)^{p_{0}-1}} \sup _{0 \leq u \leq s} \mathbb{E}\left|Y_{\Delta}(u)-D\left(Y_{\Delta}(u-\tau)\right)\right|^{p_{0}}, \forall s \geq 0 .
\end{aligned}
$$

Rearranging this gives

$$
\sup _{0 \leq u \leq s} \mathbb{E}\left|Y_{\Delta}(u)\right|^{p_{0}} \leq \frac{v}{1-v}\|\xi\|^{p_{0}}+\frac{1}{(1-v)^{p_{0}}} \sup _{0 \leq u \leq s} \mathbb{E}\left|Y_{\Delta}(u)-D\left(Y_{\Delta}(u-\tau)\right)\right|^{p_{0}}, \quad \forall s \geq 0 .
$$

105 Plugging (4.14) into 4.17) and applying the Gronwall inequality complete the proof.

Lemma 4.4. Suppose that Assumptions 2.2 and 2.4 hold with $p_{0} \geq 4 \vee 2(1+2 l)$ and $\alpha \in(0,1 / 2]$ is arbitrary. Then for any $p \in\left[2, \frac{p_{0}}{1+2 l}\right]$, the tamed EM solution $Y_{\Delta}(t)$ defined by (3.2) with modified coefficients Type II has the property that

$$
\mathbb{E} \int_{0}^{T}\left|f\left(\bar{Y}_{\Delta}(s), \bar{Y}_{\Delta}(s-\tau)\right)-f_{\Delta}\left(\bar{Y}_{\Delta}(s), \bar{Y}_{\Delta}(s-\tau)\right)\right|^{p} d s \leq C \Delta^{\alpha p}, \forall \Delta \in(0,1],
$$

and

$$
\mathbb{E} \int_{0}^{T}\left|g\left(\bar{Y}_{\Delta}(s), \bar{Y}_{\Delta}(s-\tau)\right)-g_{\Delta}\left(\bar{Y}_{\Delta}(s), \bar{Y}_{\Delta}(s-\tau)\right)\right|^{p} d s \leq C \Delta^{\alpha p}, \forall \Delta \in(0,1] .
$$


Proof. Let $\alpha \in(0,1 / 2]$ and $p \in\left[2, \frac{p_{0}}{1+2 l}\right]$. Consider the tamed EM scheme Type II. In view of Lemma 4.3, we have

$$
\begin{aligned}
& \mathbb{E} \int_{0}^{T}\left|f\left(\bar{Y}_{\Delta}(s), \bar{Y}_{\Delta}(s-\tau)\right)-f_{\Delta}\left(\bar{Y}_{\Delta}(s), \bar{Y}_{\Delta}(s-\tau)\right)\right|^{p} d s \\
& \leq \Delta^{\alpha p} \mathbb{E} \int_{0}^{T}\left[\frac{\left(\left|\bar{Y}_{\Delta}(s)\right|^{l}+\left|\bar{Y}_{\Delta}(s-\tau)\right|^{l}\right)^{p}\left|f\left(\bar{Y}_{\Delta}(s), \bar{Y}_{\Delta}(s-\tau)\right)\right|^{p}}{\left(1+\Delta^{\alpha}\left(\left|\bar{Y}_{\Delta}(s)\right|^{l}+\left|\bar{Y}_{\Delta}(s-\tau)\right|^{l}\right)\right)^{p}}\right] d s \\
& \leq C \Delta^{\alpha p} \mathbb{E} \int_{0}^{T}\left[\left(\left|\bar{Y}_{\Delta}(s)\right|^{l p}+\left|\bar{Y}_{\Delta}(s-\tau)\right|^{l p}\right)\left(1+\left|\bar{Y}_{\Delta}(s)\right|^{l+1}+\left|\bar{Y}_{\Delta}(s-\tau)\right|^{l+1}\right)^{p}\right] d s \\
& \leq C \Delta^{\alpha p} \int_{0}^{T} \mathbb{E}\left(1+\left|\bar{Y}_{\Delta}(s)\right|^{p(1+2 l)}+\left|\bar{Y}_{\Delta}(s-\tau)\right|^{p(1+2 l)}\right) d s \\
& \leq C \Delta^{\alpha p},
\end{aligned}
$$

which yields (4.18). Applying the same techniques gives 4.19).

The following lemma provides the closeness between the two continuous versions of the tamed EM solutions in the sense of $\mathcal{L}^{p}$.

Lemma 4.5. Suppose that Assumptions 2.2 and 2.4 hold with $p_{0} \geq 4 \vee(2+l)$ and $\alpha \in(0,1 / 2]$ is arbitrary. Then for any $p \in\left[2, \frac{p_{0}}{1+l / 2}\right]$, the tamed EM solution $Y_{\Delta}(t)$ defined by 3.2 with modified coefficients Type II has the property that

$$
\sup _{0 \leq t \leq T} \mathbb{E}\left|Y_{\Delta}(t)-\bar{Y}_{\Delta}(t)\right|^{p} \leq C \Delta^{(\varrho \wedge 0.5) p}, \forall \Delta \in(0,1] .
$$

Proof. Let $p \in\left[2, \frac{p_{0}}{1+l / 2}\right]$. Consider the tamed EM scheme Type II. Recall 3.6 that

$$
Y_{\Delta}(t)-\bar{Y}_{\Delta}(t)=D\left(Y_{\Delta}(t-\tau)\right)-D\left(\bar{Y}_{\Delta}(t-\tau)\right)+\varphi_{\Delta}(t), \forall t \in[0, T],
$$

where

$$
\varphi_{\Delta}(t):=\int_{\kappa(t)}^{t} f_{\Delta}\left(\bar{Y}_{\Delta}(s), \bar{Y}_{\Delta}(s-\tau)\right) d s+\int_{\kappa(t)}^{t} g_{\Delta}\left(\bar{Y}_{\Delta}(s), \bar{Y}_{\Delta}(s-\tau)\right) d B(s) .
$$

We first show that there is a positive constant $\bar{c}_{p}$ dependent of $p$ such that

$$
\mathbb{E}\left|\varphi_{\Delta}(t)\right|^{p} \leq \bar{c}_{p} \Delta^{0.5 p}, \forall t \in[0, T] .
$$

By the elementary inequality, we have that for any $t \in[0, T]$

$$
\begin{aligned}
\mathbb{E}\left|\varphi_{\Delta}(t)\right|^{p} & =\mathbb{E}\left|\int_{\kappa(t)}^{t} f_{\Delta}\left(\bar{Y}_{\Delta}(s), \bar{Y}_{\Delta}(s-\tau)\right) d s+\int_{\kappa(t)}^{t} g_{\Delta}\left(\bar{Y}_{\Delta}(s), \bar{Y}_{\Delta}(s-\tau)\right) d B(s)\right|^{p} \\
& \leq 2^{p-1} \Delta^{p-1} \mathbb{E} \int_{\kappa(t)}^{t}\left|f_{\Delta}\left(\bar{Y}_{\Delta}(s), \bar{Y}_{\Delta}(s-\tau)\right)\right|^{p} d s+2^{p-1} \mathbb{E}\left|\int_{\kappa(t)}^{t} g_{\Delta}\left(\bar{Y}_{\Delta}(s), \bar{Y}_{\Delta}(s-\tau)\right) d B(s)\right|^{p} .
\end{aligned}
$$


On the basis of the Hölder inequality, Lemma 4.3 and P2, we have

$$
\begin{aligned}
2^{p-1} \Delta^{p-1} \mathbb{E} \int_{\kappa(t)}^{t}\left|f_{\Delta}\left(\bar{Y}_{\Delta}(s), \bar{Y}_{\Delta}(s-\tau)\right)\right|^{p} d s & \leq C \Delta^{p-1} \mathbb{E} \int_{\kappa(t)}^{t}\left[\Delta^{-p \alpha}\left(1+\left|\bar{Y}_{\Delta}(s)\right|^{p}+|\bar{Y}(s-\tau)|^{p}\right)\right] d s \\
& \leq C \Delta^{p(1-\alpha)}
\end{aligned}
$$

By the Burkholder-Davis-Gundy inequality, P2 and 2.8, we have

$$
\begin{aligned}
\mathbb{E}\left|\int_{\kappa(t)}^{t} g_{\Delta}\left(\bar{Y}_{\Delta}(s), \bar{Y}_{\Delta}(s-\tau)\right) d B(s)\right|^{p} & \leq\left.\left. C \mathbb{E}\left|\int_{\kappa(t)}^{t}\right| g_{\Delta}\left(\bar{Y}_{\Delta}(s), \bar{Y}_{\Delta}(s-\tau)\right)\right|^{2} d s\right|^{p / 2} \\
& \leq C \mathbb{E}\left(\int_{\kappa(t)}^{t}\left(1+\left|\bar{Y}_{\Delta}(s)\right|^{l+2}+\left|\bar{Y}_{\Delta}(s-\tau)\right|^{l+2}\right) d s\right)^{p / 2} \\
& \leq C \mathbb{E}\left(\left(1+\left|Y_{\Delta}(\kappa(t))\right|^{l+2}+\left|Y_{\Delta}(\kappa(t)-\tau)\right|^{l+2}\right) \Delta\right)^{p / 2} \\
& \leq C \Delta^{p / 2}\left(1+\sup _{0 \leq s \leq t} \mathbb{E}\left|Y_{\Delta}(s)\right|^{p(l / 2+1)}\right) \leq C \Delta^{p / 2}
\end{aligned}
$$

Noting that $\alpha \in(0,1 / 2]$, from 4.23$)$-4.25), we get 4.22 . On the other hand, by 4.15 and 2.3 , we see from 4.21, that

$$
\begin{aligned}
\left|Y_{\Delta}(t)-\bar{Y}_{\Delta}(t)\right|^{p} & =\left|D\left(Y_{\Delta}(t-\tau)\right)-D\left(\bar{Y}_{\Delta}(t-\tau)\right)+\varphi_{\Delta}(t)\right|^{p} \\
& \leq\left(1+\varepsilon^{\frac{1}{p-1}}\right)^{p-1}\left(\frac{\left|D\left(Y_{\Delta}(t-\tau)\right)-D\left(\bar{Y}_{\Delta}(t-\tau)\right)\right|^{p}}{\varepsilon}+\left|\varphi_{\Delta}(t)\right|^{p}\right) \\
& \leq\left(1+\varepsilon^{\frac{1}{p-1}}\right)^{p-1}\left(\frac{v^{p}\left|Y_{\Delta}(t-\tau)-\bar{Y}_{\Delta}(t-\tau)\right|^{p}}{\varepsilon}+\left|\varphi_{\Delta}(t)\right|^{p}\right), \forall t \in[0, T] .
\end{aligned}
$$

Letting $\varepsilon=\left(\frac{v}{1-v}\right)^{p-1}$ gives that

$$
\left|Y_{\Delta}(t)-\bar{Y}_{\Delta}(t)\right|^{p} \leq v\left|Y_{\Delta}(t-\tau)-\bar{Y}_{\Delta}(t-\tau)\right|^{p}+\frac{1}{(1-v)^{p-1}}\left|\varphi_{\Delta}(t)\right|^{p}, \forall t \in[0, T] .
$$

Taking expectations on the both sides of 4.26 and using 4.22 , 2.2), we have

$$
\begin{aligned}
\mathbb{E}\left|Y_{\Delta}(t)-\bar{Y}_{\Delta}(t)\right|^{p} & \leq v \mathbb{E}\left|Y_{\Delta}(t-\tau)-\bar{Y}_{\Delta}(t-\tau)\right|^{p}+\frac{1}{(1-v)^{p-1}} \mathbb{E}\left|\varphi_{\Delta}(t)\right|^{p} \\
& \leq v \sup _{-\tau \leq t \leq T} \mathbb{E}\left|Y_{\Delta}(t)-\bar{Y}_{\Delta}(t)\right|^{p}+\frac{\bar{c}_{p}}{(1-v)^{p-1}} \Delta^{0.5 p} \\
& \leq v \sup _{0 \leq t \leq T} \mathbb{E}\left|Y_{\Delta}(t)-\bar{Y}_{\Delta}(t)\right|^{p}+v K_{1}^{p} \Delta^{\varrho p}+\frac{\bar{c}_{p}}{(1-v)^{p-1}} \Delta^{0.5 p} .
\end{aligned}
$$

As this holds for any $t \in[0, T]$, thus

$$
\sup _{0 \leq t \leq T} \mathbb{E}\left|Y_{\Delta}(t)-\bar{Y}_{\Delta}(t)\right|^{p} \leq v \sup _{0 \leq t \leq T} \mathbb{E}\left|Y_{\Delta}(t)-\bar{Y}_{\Delta}(t)\right|^{p}+\left(v K_{1}^{p}+\frac{\bar{c}_{p}}{(1-v)^{p-1}}\right) \Delta^{(\varrho \wedge 0.5) p}
$$

Rearranging this implies the desired assertion. 
Proof of Theorem 4.1. Consider the tamed EM solution $Y_{\Delta}(t)$ with modified coefficients Type II. Denote

$$
e_{\Delta}(t):=x(t)-Y_{\Delta}(t)-D(x(t-\tau))+D\left(Y_{\Delta}(t-\tau)\right), \forall t \in[0, T] .
$$

Thus, by (2.3) and the elementary inequality, we have that for any $t \in[0, T]$,

$$
\begin{aligned}
\left|x(t)-Y_{\Delta}(t)\right|^{2} & \leq(1+\varepsilon)\left|D(x(t-\tau))-D\left(Y_{\Delta}(t-\tau)\right)\right|^{2}+\left(1+\varepsilon^{-1}\right)\left|e_{\Delta}(t)\right|^{2} \\
& \leq(1+\varepsilon) v^{2}\left|x(t-\tau)-Y_{\Delta}(t-\tau)\right|^{2}+\left(1+\varepsilon^{-1}\right)\left|e_{\Delta}(t)\right|^{2} .
\end{aligned}
$$

Note that $x(t)=Y_{\Delta}(t)$ for any $t \in[-\tau, 0]$. Then letting $\varepsilon=\frac{1-v}{v}$ and taking expectations on the both sides of 4.30, we have

$$
\begin{aligned}
\sup _{0 \leq u \leq t} \mathbb{E}\left|x(u)-Y_{\Delta}(u)\right|^{2} & \leq v \sup _{0 \leq u \leq t} \mathbb{E}\left|x(u-\tau)-Y_{\Delta}(u-\tau)\right|^{2}+\frac{1}{1-v} \sup _{0 \leq u \leq t} \mathbb{E}\left|e_{\Delta}(u)\right|^{2} \\
& \leq v \sup _{0 \leq u \leq t} \mathbb{E}\left|x(u)-Y_{\Delta}(u)\right|^{2}+\frac{1}{1-v} \sup _{0 \leq u \leq t} \mathbb{E}\left|e_{\Delta}(u)\right|^{2}, \quad \forall t \in[0, T] .
\end{aligned}
$$

Rearranging this gives

$$
\sup _{0 \leq u \leq t} \mathbb{E}\left|x(u)-Y_{\Delta}(u)\right|^{2} \leq \frac{1}{(1-v)^{2}} \sup _{0 \leq u \leq t} \mathbb{E}\left|e_{\Delta}(u)\right|^{2}, \forall t \in[0, T] .
$$

By the Itô formula and the elementary inequality, we have that for any $t \in[0, T]$

$$
\begin{aligned}
\mathbb{E}\left|e_{\Delta}(t)\right|^{2}= & \mathbb{E} \int_{0}^{t}\left[2 e_{\Delta}^{T}(s)\left[f(x(s), x(s-\tau))-f_{\Delta}\left(\bar{Y}_{\Delta}(s), \bar{Y}_{\Delta}(s-\tau)\right)\right]\right. \\
& \left.+\left|g(x(s), x(s-\tau))-g_{\Delta}\left(\bar{Y}_{\Delta}(s), \bar{Y}_{\Delta}(s-\tau)\right)\right|^{2}\right] d s \\
\leq & \mathbb{E} \int_{0}^{t}\left[2 e_{\Delta}^{T}(s)\left[f(x(s), x(s-\tau))-f\left(Y_{\Delta}(s), Y_{\Delta}(s-\tau)\right)\right]\right. \\
& \left.+\left(p_{1}-1\right)\left|g(x(s), x(s-\tau))-g\left(Y_{\Delta}(s), Y_{\Delta}(s-\tau)\right)\right|^{2}\right] d s \\
& +\mathbb{E} \int_{0}^{t} 2 e_{\Delta}^{T}(s)\left[f\left(Y_{\Delta}(s), Y_{\Delta}(s-\tau)\right)-f_{\Delta}\left(\bar{Y}_{\Delta}(s), \bar{Y}_{\Delta}(s-\tau)\right)\right] d s \\
& +\frac{p_{1}-1}{p_{1}-2} \mathbb{E} \int_{0}^{t}\left|g\left(Y_{\Delta}(s), Y_{\Delta}(s-\tau)\right)-g_{\Delta}\left(\bar{Y}_{\Delta}(s), \bar{Y}_{\Delta}(s-\tau)\right)\right|^{2} d s .
\end{aligned}
$$

By Assumption 2.4, we have

$$
\mathbb{E}\left|e_{\Delta}(t)\right|^{2} \leq I_{1}(t)+I_{2}(t),
$$

where

$$
\begin{aligned}
I_{1}(t):= & \mathbb{E} \int_{0}^{t}\left[\left|e_{\Delta}(s)\right|^{2}+2 K_{3}\left(\left|x(s)-Y_{\Delta}(s)\right|^{2}+\left|x(s-\tau)-Y_{\Delta}(s-\tau)\right|^{2}\right)\right. \\
& \left.-2 U\left(x(s), Y_{\Delta}(s)\right)+2 U\left(x(s-\tau), Y_{\Delta}(s-\tau)\right)\right] d s
\end{aligned}
$$


and

$$
\begin{aligned}
I_{2}(t):= & \mathbb{E} \int_{0}^{t}\left[\left|f\left(Y_{\Delta}(s), Y_{\Delta}(s-\tau)\right)-f_{\Delta}\left(\bar{Y}_{\Delta}(s), \bar{Y}_{\Delta}(s-\tau)\right)\right|^{2}\right. \\
& \left.+\frac{p_{1}-1}{p_{1}-2}\left|g\left(Y_{\Delta}(s), Y_{\Delta}(s-\tau)\right)-g_{\Delta}\left(\bar{Y}_{\Delta}(s), \bar{Y}_{\Delta}(s-\tau)\right)\right|^{2}\right] d s .
\end{aligned}
$$

Recalling that for any $s \in[-\tau, 0], x(s)=Y_{\Delta}(s)$ and $U\left(x(s), Y_{\Delta}(s)\right)=0$, we have

$$
\int_{0}^{t}\left|x(s-\tau)-Y_{\Delta}(s-\tau)\right|^{2} d s \leq \int_{0}^{t}\left|x(s)-Y_{\Delta}(s)\right|^{2} d s,
$$

and

$$
\int_{0}^{t} U\left(x(s-\tau), Y_{\Delta}(s-\tau)\right) d s \leq \int_{-\tau}^{t} U\left(x(s), Y_{\Delta}(s)\right) d s=\int_{0}^{t} U\left(x(s), Y_{\Delta}(s)\right) d s,
$$

as well as

$$
\left|e_{\Delta}(s)\right|^{2} \leq 2\left|x(s)-Y_{\Delta}(s)\right|^{2}+2 v^{2}\left|x(s-\tau)-Y_{\Delta}(s-\tau)\right|^{2}, \forall s \in[0, t] .
$$

Inserting (4.36) into $I_{1}(t)$ and using 4.34), 4.35), we have

$$
I_{1}(t) \leq\left(4 K_{3}+2+2 v^{2}\right) \int_{0}^{t} \mathbb{E}\left|x(s)-Y_{\Delta}(s)\right|^{2} d s .
$$

To estimate $I_{2}(t)$, we observe that

$$
I_{2}(t) \leq I_{21}(t)+I_{22}(t)
$$

where

$$
\begin{aligned}
I_{21}(t):= & 2 \int_{0}^{T} \mathbb{E}\left|f\left(Y_{\Delta}(s), Y_{\Delta}(s-\tau)\right)-f\left(\bar{Y}_{\Delta}(s), \bar{Y}_{\Delta}(s-\tau)\right)\right|^{2} d s \\
& +\frac{2\left(p_{1}-1\right)}{p_{1}-2} \int_{0}^{T} \mathbb{E}\left|g\left(Y_{\Delta}(s), Y_{\Delta}(s-\tau)\right)-g\left(\bar{Y}_{\Delta}(s), \bar{Y}_{\Delta}(s-\tau)\right)\right|^{2} d s,
\end{aligned}
$$

and

$$
\begin{aligned}
I_{22}(t):= & 2 \int_{0}^{T} \mathbb{E}\left|f\left(\bar{Y}_{\Delta}(s), \bar{Y}_{\Delta}(s-\tau)\right)-f_{\Delta}\left(\bar{Y}_{\Delta}(s), \bar{Y}_{\Delta}(s-\tau)\right)\right|^{2} d s \\
& +\frac{2\left(p_{1}-1\right)}{p_{1}-2} \int_{0}^{T} \mathbb{E}\left|g\left(\bar{Y}_{\Delta}(s), \bar{Y}_{\Delta}(s-\tau)\right)-g_{\Delta}\left(\bar{Y}_{\Delta}(s), \bar{Y}_{\Delta}(s-\tau)\right)\right|^{2} d s .
\end{aligned}
$$

By the condition $p_{0} \geq 4 \vee 2(1+2 l)$, we have

$$
\frac{2 p_{0}}{p_{0}-2 l} \leq \frac{2 p_{0}}{2+2 l}<\frac{p_{0}}{1+l / 2}
$$

Thus, according to Lemma 4.4 , we have

$$
I_{22}(t) \leq C \Delta^{2 \alpha},
$$


and applying the Hölder inequality, Lemmas 4.3 and 4.5, as well as Assumption 2.4 we have that for any $s \in[0, T]$

$$
\begin{aligned}
\mathbb{E} \mid & f\left(Y_{\Delta}(s), Y_{\Delta}(s-\tau)\right)-\left.f\left(\bar{Y}_{\Delta}(s), \bar{Y}_{\Delta}(s-\tau)\right)\right|^{2} \\
\leq & C \mathbb{E}\left[\left(1+\left|Y_{\Delta}(s)\right|^{2 l}+\left|Y_{\Delta}(s-\tau)\right|^{2 l}+\left|\bar{Y}_{\Delta}(s)\right|^{2 l}+\left|\bar{Y}_{\Delta}(s-\tau)\right|^{2 l}\right)\right. \\
& \left.\times\left(\left|Y_{\Delta}(s)-\bar{Y}_{\Delta}(s)\right|^{2}+\left|Y_{\Delta}(s-\tau)-\bar{Y}_{\Delta}(s-\tau)\right|^{2}\right)\right] \\
\leq & C\left(1+\mathbb{E}\left|Y_{\Delta}(s)\right|^{p_{0}}+\mathbb{E}\left|Y_{\Delta}(s-\tau)\right|^{p_{0}}+\mathbb{E}\left|\bar{Y}_{\Delta}(s)\right|^{p_{0}}+\mathbb{E}\left|\bar{Y}_{\Delta}(s-\tau)\right|^{p_{0}}\right)^{2 l / p_{0}} \\
& \times\left(\mathbb{E}\left|Y_{\Delta}(s)-\bar{Y}_{\Delta}(s)\right|^{2 p_{0} /\left(p_{0}-2 l\right)}+\mathbb{E}\left|Y_{\Delta}(s-\tau)-\bar{Y}_{\Delta}(s-\tau)\right|^{2 p_{0} /\left(p_{0}-2 l\right)}\right)^{\left(p_{0}-2 l\right) / p_{0}} \\
\leq & C \Delta^{2 \varrho \wedge 1} .
\end{aligned}
$$

Similarly, we can deduce that

$$
\mathbb{E}\left|g\left(Y_{\Delta}(s)-Y_{\Delta}(s-\tau)\right)-g\left(\bar{Y}_{\Delta}(s), \bar{Y}_{\Delta}(s-\tau)\right)\right|^{2} \leq C \Delta^{2 \varrho \wedge 1} .
$$

Noting that $\alpha \in(0,1 / 2]$, combining (4.37), 4.38), 4.39) and (4.40) together, we observe from 4.33 that

$$
\mathbb{E}\left|e_{\Delta}(t)\right|^{2} \leq C \int_{0}^{t} \mathbb{E}\left|x(s)-Y_{\Delta}(s)\right|^{2} d s+C \Delta^{2(\alpha \wedge \varrho)} .
$$

As this holds for any $t \in[0, T]$, thus

$$
\sup _{0 \leq u \leq t} \mathbb{E}\left|e_{\Delta}(u)\right|^{2} \leq C \int_{0}^{t} \sup _{0 \leq u \leq s} \mathbb{E}\left|x(u)-Y_{\Delta}(u)\right|^{2} d s+C \Delta^{2(\alpha \wedge \varrho)} .
$$

Substituting this into 4.31 gets

$$
\sup _{0 \leq u \leq t} \mathbb{E}\left|x(u)-Y_{\Delta}(u)\right|^{2} \leq C \int_{0}^{t} \sup _{0 \leq u \leq s} \mathbb{E}\left|x(u)-Y_{\Delta}(u)\right|^{2} d s+C \Delta^{2(\alpha \wedge \varrho)} .
$$

110 Applying the Gronwall inequality give the first assertion in 4.1. Combining this with Lemma 4.5 yields the second in 4.1. Thus, the proof is complete.

\subsection{Strong convergence (without order) of tamed EM (Type I) under local Lipschitz condition}

In this section, we mainly discuss the convergence issue of tamed EM scheme when global monotonicity and polynomial growth conditions are replaced by the local Lipschitz condition.

115 Note that Lemma 4.3 requires the condition $p_{0} \geq 4$, however, if we are only concerned with the convergence (without order) of the tamed EM scheme, then this condition can be substituted by a more relaxed $p_{0}>2$. The price we pay for this is a narrow scope of the parameter $\alpha$, which will lead to a decrease in the convergence rate in view of Theorem 4.1 Borrowing the method of the proof of moments boundedness in Mao [6, Lemma 3.2], we have the following lemma.

Lemma 4.6. Suppose that Assumptions 2.1 and 2.2 hold with $p_{0}>2$ and $\alpha \in(0,1 / 3]$ is arbitrary. Then the tamed EM solution $Y_{\Delta}(t)$ given by (3.2) with modified coefficients Type I satisfies

$$
\sup _{0<\Delta \leq 1} \sup _{0 \leq t \leq T} \mathbb{E}\left|Y_{\Delta}(t)\right|^{p_{0}} \leq C,
$$

where the positive constant $C:=C\left(p_{0}, T, K_{2},\|\xi\|, v\right)$. 
Proof. Let Assumptions 2.1 and 2.2 hold with $p_{0}>2$. Consider the tamed EM scheme Type I. Let us begin with an assertion that for any $\hat{p}>0$,

$$
\begin{aligned}
& \mathbb{E}\left[\left.\left|Y_{\Delta}(t)-\bar{Y}_{\Delta}(t)-D\left(Y_{\Delta}(t-\tau)\right)+D\left(\bar{Y}_{\Delta}(t-\tau)\right)\right|\right|^{\hat{p}} \mid \mathcal{F}_{\kappa(t)}\right] \\
& \leq C \Delta^{\hat{p}(1-\alpha) / 2}\left(1+\left|\bar{Y}_{\Delta}(t)\right|^{\hat{p}}+\left|\bar{Y}_{\Delta}(t-\tau)\right|^{\hat{p}}\right), \forall t \geq 0,
\end{aligned}
$$

where $C$ is a positive constant independent of $\Delta$. Recall that

$$
Y_{\Delta}(t)-\bar{Y}_{\Delta}(t)-D\left(Y_{\Delta}(t-\tau)\right)+D\left(\bar{Y}_{\Delta}(t-\tau)\right)=\varphi_{\Delta}(t), \forall t \geq 0,
$$

where $\varphi_{\Delta}(t)$ is defined in 4.21], namely

$$
\varphi_{\Delta}(t)=f_{\Delta}\left(Y_{\Delta}(\kappa(t)), Y_{\Delta}(\kappa(t)-\tau)\right)(t-\kappa(t))+g_{\Delta}\left(Y_{\Delta}(\kappa(t)), Y_{\Delta}(\kappa(t)-\tau)\right)(B(t)-B(\kappa(t))) .
$$

Then for any $\hat{p} \geq 2$, using $\mathrm{P} 2$ gives

$$
\begin{aligned}
\mathbb{E}\left[\left|\varphi_{\Delta}(t)\right|^{\hat{p}} \mid \mathcal{F}_{\kappa(t)}\right] \leq & C \mathbb{E}\left[\left|f_{\Delta}\left(Y_{\Delta}(\kappa(t)), Y_{\Delta}(\kappa(t)-\tau)\right)(t-\kappa(t))\right|^{\hat{p}} \mid \mathcal{F}_{\kappa(t)}\right] \\
& +C \mathbb{E}\left[\left.\left|g_{\Delta}\left(Y_{\Delta}(\kappa(t)), Y_{\Delta}(\kappa(t)-\tau)\right)(B(t)-B(\kappa(t)))\right|\right|^{\hat{p}} \mid \mathcal{F}_{\kappa(t)}\right] \\
\leq & C \Delta^{\hat{p}(1-\alpha)}\left(1+\left|Y_{\Delta}(\kappa(t))\right|^{\hat{p}}+\left|Y_{\Delta}(\kappa(t)-\tau)\right|^{\hat{p}}\right) \\
& +C \Delta^{\hat{p}(1-\alpha) / 2}\left(1+\left|Y_{\Delta}(\kappa(t))\right|^{\hat{p}}+\left|Y_{\Delta}(\kappa(t)-\tau)\right|^{\hat{p}}\right) \\
\leq & C \Delta^{\hat{p}(1-\alpha) / 2}\left(1+\left|\bar{Y}_{\Delta}(t)\right|^{\hat{p}}+\left|\bar{Y}_{\Delta}(t-\tau)\right|^{\hat{p}}\right),
\end{aligned}
$$

this also holds for any $0<\hat{p}<2$ due to the Hölder inequality. Thus, we get the assertion (4.41). Now recall 4.5 , that

$$
\mathbb{E}\left|Y_{\Delta}(t)-D\left(Y_{\Delta}(t-\tau)\right)\right|^{p_{0}} \leq C+C \int_{0}^{t}\left[\|\xi\|^{p_{0}}+\sup _{0 \leq u \leq s} \mathbb{E}\left|Y_{\Delta}(u)\right|^{p_{0}}\right] d s+p_{0} J(t), t \geq 0,
$$

with $J(t)$ defined in 4.3 , i.e.,

$$
\begin{aligned}
J(t)= & \mathbb{E} \int_{0}^{t}\left[\left|Y_{\Delta}(s)-D\left(Y_{\Delta}(s-\tau)\right)\right|^{p_{0}-2}\right. \\
& \left.\times\left(Y_{\Delta}(s)-\bar{Y}_{\Delta}(s)-D\left(Y_{\Delta}(s-\tau)\right)+D\left(\bar{Y}_{\Delta}(s-\tau)\right)\right)^{T} f_{\Delta}\left(\bar{Y}_{\Delta}(s), \bar{Y}_{\Delta}(s-\tau)\right)\right] d s .
\end{aligned}
$$

By the Young inequality, we have

$$
J(t) \leq \frac{p_{0}-2}{p_{0}} \mathbb{E} \int_{0}^{t}\left|Y_{\Delta}(s)-D\left(Y_{\Delta}(s-\tau)\right)\right|^{p_{0}} d s+\frac{2}{p_{0}} \Pi(t),
$$

where

$$
\Pi(t)=\mathbb{E} \int_{0}^{t}\left|Y_{\Delta}(s)-\bar{Y}_{\Delta}(s)-D\left(Y_{\Delta}(s)\right)+D\left(\bar{Y}_{\Delta}(s-\tau)\right)\right|^{p_{0} / 2}\left|f_{\Delta}\left(\bar{Y}_{\Delta}(s), \bar{Y}_{\Delta}(s-\tau)\right)\right|^{p_{0} / 2} d s .
$$

Let $\alpha \in(0,1 / 3$ ], using (4.41) and $\mathrm{P} 2$ as well as 4.4) yields

$$
\begin{aligned}
\Pi(t) & \left.\left.\leq\left. C \Delta^{(1-\alpha) p_{0} / 4-\alpha p_{0} / 2} \mathbb{E} \int_{0}^{t}\left(1+\mid \bar{Y}_{\Delta}(s)\right)\right|^{p_{0} / 2}+\mid \bar{Y}_{\Delta}(s-\tau)\right)\left.\right|^{p_{0} / 2}\right)^{2} d s \\
& \leq C \Delta^{(1-3 \alpha) p_{0} / 4} \int_{0}^{t}\left[1+\sup _{0 \leq u \leq s} \mathbb{E}\left|\bar{Y}_{\Delta}(u)\right|^{p_{0}}+\sup _{0 \leq u \leq s} \mathbb{E}\left|\bar{Y}_{\Delta}(u-\tau)\right|^{p_{0}}\right] d s \\
& \leq C \int_{0}^{t}\left[1+\|\xi\|^{p_{0}}+\sup _{0 \leq u \leq s} \mathbb{E}\left|Y_{\Delta}(u)\right|^{p_{0}}\right] d s .
\end{aligned}
$$


We observe from (4.44) and 4.45 as well as 4.46) that

$$
\sup _{0 \leq u \leq t} \mathbb{E}\left|Y_{\Delta}(u)-D\left(Y_{\Delta}(u-\tau)\right)\right|^{p_{0}} \leq C+C \int_{0}^{t} \sup _{0 \leq u \leq s} \mathbb{E}\left|Y_{\Delta}(u)\right|^{p_{0}} d s .
$$

Inserting this into (4.17) and using the Gronwall inequality give the desired assertion.

Lemma 4.7. Suppose that Assumptions 2.1 and 2.2 hold with $p_{0}>2$ and $\alpha \in(0,1 / 3$ ] is arbitrary. Consider the tamed EM solution $Y_{\Delta}(t)$ defined by (3.2) with modified coefficients Type I. For any real number $R>\|\xi\|$ and $\Delta \in(0,1]$, define the stoping time $\bar{\rho}_{\Delta, R}=\inf \left\{t \geq 0:\left|Y_{\Delta}(t)\right| \geq R\right\}$. Then

$$
\mathbb{P}\left(\bar{\rho}_{\Delta, R} \leq T\right) \leq \frac{C}{R^{2}},
$$

where $C$ is a positive constant independent of $\Delta$ and $R$.

Proof. Write $\bar{\rho}_{\Delta, R}=\bar{\rho}$ for short. By the Itô formula and 3.13, we have

$$
\begin{aligned}
& \mathbb{E}\left|Y_{\Delta}(T \wedge \bar{\rho})-D\left(Y_{\Delta}(T \wedge \bar{\rho}-\tau)\right)\right|^{2}-|\xi(0)-D(\xi(-\tau))|^{2} \\
& =\mathbb{E} \int_{0}^{T \wedge \bar{\rho}}\left[2\left(Y_{\Delta}(s)-D\left(Y_{\Delta}(s-\tau)\right)\right)^{T} f_{\Delta}\left(\bar{Y}_{\Delta}(s), \bar{Y}_{\Delta}(s-\tau)\right)+\left|g_{\Delta}\left(\bar{Y}_{\Delta}(s), \bar{Y}_{\Delta}(s-\tau)\right)\right|^{2}\right] d s \\
& =\mathbb{E} \int_{0}^{T \wedge \bar{\rho}}\left[2\left(\bar{Y}_{\Delta}(s)-D\left(\bar{Y}_{\Delta}(s-\tau)\right)\right)^{T} f_{\Delta}\left(\bar{Y}_{\Delta}(s), \bar{Y}_{\Delta}(s-\tau)\right)+\left|g_{\Delta}\left(\bar{Y}_{\Delta}(s), \bar{Y}_{\Delta}(s-\tau)\right)\right|^{2}\right] d s+2 I^{\star}(T) \\
& \leq 2 \hat{K}_{2} \mathbb{E} \int_{0}^{T \wedge \bar{\rho}}\left(1+\left|\bar{Y}_{\Delta}(s)\right|^{2}+\left|\bar{Y}_{\Delta}(s-\tau)\right|^{2}\right) d s+2 I^{\star}(T) \\
& \leq 2 \hat{K}_{2} \int_{0}^{T} \mathbb{E}\left(1+\left|\bar{Y}_{\Delta}(s)\right|^{2}+\left|\bar{Y}_{\Delta}(s-\tau)\right|^{2}\right) d s+2 I^{\star}(T)
\end{aligned}
$$

where

$$
I^{\star}(T)=\mathbb{E} \int_{0}^{T \wedge \bar{\rho}}\left[\left(Y_{\Delta}(s)-\bar{Y}_{\Delta}(s)-D\left(Y_{\Delta}(s-\tau)\right)+D\left(\bar{Y}_{\Delta}(s-\tau)\right)\right)^{T} f_{\Delta}\left(\bar{Y}_{\Delta}(s), \bar{Y}_{\Delta}(s-\tau)\right)\right] d s .
$$

By 3.11 and 4.41 as well as the condition that $\alpha \in(0,1 / 3]$, we have

$$
\begin{aligned}
I^{\star}(T) & \leq \int_{0}^{T} \mathbb{E}\left[\left|Y_{\Delta}(s)-\bar{Y}_{\Delta}(s)-D\left(Y_{\Delta}(s-\tau)\right)+D\left(\bar{Y}_{\Delta}(s-\tau)\right) \| f_{\Delta}\left(\bar{Y}_{\Delta}(s), \bar{Y}_{\Delta}(s-\tau)\right)\right|\right] d s \\
& =\int_{0}^{T} \mathbb{E}\left(\left|f_{\Delta}\left(\bar{Y}_{\Delta}(s), \bar{Y}_{\Delta}(s-\tau)\right)\right| \mathbb{E}\left[\left|Y_{\Delta}(s)-\bar{Y}_{\Delta}(s)-D\left(Y_{\Delta}(s-\tau)\right)+D\left(\bar{Y}_{\Delta}(s-\tau)\right)\right| \mid \mathcal{F}_{\kappa(s)}\right]\right) d s \\
& \leq C \Delta^{(1-3 \alpha) / 2} \int_{0}^{T} \mathbb{E}\left(1+\left|\bar{Y}_{\Delta}(s)\right|^{2}+\left|\bar{Y}_{\Delta}(s-\tau)\right|^{2}\right) d s \\
& \leq C \int_{0}^{T} \mathbb{E}\left(1+\left|\bar{Y}_{\Delta}(s)\right|^{2}+\left|\bar{Y}_{\Delta}(s-\tau)\right|^{2}\right) d s .
\end{aligned}
$$

Inserting this into 4.47 and using Lemma 4.6 give

$$
\mathbb{E}\left|Y_{\Delta}(T \wedge \bar{\rho})-D\left(Y_{\Delta}(T \wedge \bar{\rho}-\tau)\right)\right|^{2} \leq C .
$$


Note that

$$
\begin{aligned}
& \mathbb{I}_{\{\bar{\rho} \leq T\}}\left|Y_{\Delta}(\bar{\rho})-D\left(Y_{\Delta}(\bar{\rho}-\tau)\right)\right| \geq \mathbb{I}_{\{\bar{\rho} \leq T\}}\left(\left|Y_{\Delta}(\bar{\rho})\right|-\left|D\left(Y_{\Delta}(\bar{\rho}-\tau)\right)\right|\right) \\
& \geq R-v \mathbb{I}_{\{\bar{\rho} \leq T\}}\left|Y_{\Delta}(\bar{\rho}-\tau)\right| \geq R-v R=(1-v) R .
\end{aligned}
$$

Thus, from 4.49 and 4.50, we get

$$
\begin{aligned}
\mathbb{P}(\bar{\rho} \leq T) & \leq \frac{\mathbb{E}\left[\mathbb{I}_{\{\bar{\rho} \leq T\}}\left|Y_{\Delta}(\bar{\rho})-D\left(Y_{\Delta}(\bar{\rho}-\tau)\right)\right|^{2}\right]}{(1-v)^{2} R^{2}} \\
& \leq \frac{\mathbb{E}\left[\left|Y_{\Delta}(T \wedge \bar{\rho})-D\left(Y_{\Delta}(T \wedge \bar{\rho}-\tau)\right)\right|^{2}\right]}{(1-v)^{2} R^{2}} \\
& \leq \frac{C}{(1-v)^{2} R^{2}},
\end{aligned}
$$

which gives the desired assertion.

Similarly, we can show the following lemma.

Lemma 4.8. Suppose that Assumptions 2.1 and 2.2 hold with $p_{0}>2$. For any real number $R>\|\xi\|$, define the stoping time $\rho_{R}=\inf \{t \geq 0:|x(t)| \geq R\}$. Then

$$
\mathbb{P}\left(\rho_{R} \leq T\right) \leq \frac{C}{R^{2}}
$$

where $C$ is a positive constant independent of $R$.

Theorem 4.9. Suppose that Assumptions 2.1 and 2.2 hold with $p_{0}>2$ and $\alpha \in(0,1 / 3]$ is arbitrary. Consider the tamed EM scheme Type I. Then for any $q \in\left[2, p_{0}\right)$,

$$
\lim _{\Delta \rightarrow 0} \mathbb{E}\left|x(T)-Y_{\Delta}(T)\right|^{q}=0 \quad \text { and } \quad \lim _{\Delta \rightarrow 0} \mathbb{E}\left|x(T)-\bar{Y}_{\Delta}(T)\right|^{q}=0
$$

Proof. For any $R>\|\xi\|$, denote $\theta_{\Delta, R}:=\rho_{R} \wedge \bar{\rho}_{\Delta, R}$ and $e_{\Delta}^{\star}(t):=x(t)-Y_{\Delta}(t)$, recall $e_{\Delta}(t)=$ $x(t)-D(x(t-\tau))-Y_{\Delta}(t)+D\left(Y_{\Delta}(t-\tau)\right)$. Then for any $q \in\left[2, p_{0}\right)$ and $\eta>0$, the Young inequality gives

$$
\begin{aligned}
\mathbb{E}\left|e_{\Delta}^{\star}(T)\right|^{q} & =\mathbb{E}\left[\left|e_{\Delta}^{\star}(T)\right|^{q} \mathbb{I}_{\left\{\theta_{\Delta, R}>T\right\}}\right]+\mathbb{E}\left[\left|e_{\Delta}^{\star}(T)\right|^{q} \mathbb{I}_{\left\{\theta_{\Delta, R} \leq T\right\}}\right] \\
& \leq \mathbb{E}\left[\left|e_{\Delta}^{\star}(T)\right|^{q} \mathbb{I}_{\left\{\theta_{\Delta, R}>T\right\}}\right]+\frac{q \eta}{p_{0}} \mathbb{E}\left|e_{\Delta}^{\star}(T)\right|^{q}+\frac{p_{0}-q}{p_{0} \eta^{q /\left(p_{0}-q\right)}} \mathbb{P}\left(\theta_{\Delta, R} \leq T\right) .
\end{aligned}
$$

In this subsection, $C_{R}$ denotes a positive constant depending on $R$, its value may be different for different appearance. By Lemmas 2.3 and 4.6 , we have

$$
\mathbb{E}\left|e_{\Delta}^{\star}(T)\right|^{q} \leq 2^{q-1}\left(\mathbb{E}|x(T)|^{q}+\mathbb{E}\left|Y_{\Delta}(T)\right|^{q}\right) \leq C .
$$

While by Lemmas 4.7 and 4.8

$$
\mathbb{P}\left(\theta_{\Delta, R} \leq T\right) \leq \mathbb{P}\left(\rho_{R} \leq T\right)+\mathbb{P}\left(\bar{\rho}_{\Delta, R} \leq T\right) \leq \frac{C}{R^{2}}
$$


Plugging (4.54) and 4.55) into 4.53, we get

$$
\mathbb{E}\left|e_{\Delta}^{\star}(T)\right|^{q} \leq \mathbb{E}\left[\left|e_{\Delta}^{\star}(T)\right|^{q} \mathbb{I}_{\left\{\theta_{\Delta, R}>T\right\}}\right]+\frac{C q \eta}{p_{0}}+\frac{C\left(p_{0}-q\right)}{p_{0} R^{2} \eta^{q /\left(p_{0}-q\right)}} .
$$

Next, we shall prove that for any $R>0$, there exist a positive constant $C_{R}$ such that

$$
\mathbb{E}\left|e_{\Delta}^{\star}\left(T \wedge \theta_{\Delta, R}\right)\right|^{q} \leq C_{R} \Delta^{(\alpha \wedge \varrho) q} .
$$

In the same way as Lemma 4.5 was proved, applying Assumption 2.1 and P2, we can show that

$$
\sup _{0 \leq t \leq T} \mathbb{E}|Y(t)-\bar{Y}(t)|^{q} \leq C_{R} \Delta^{q / 2} .
$$

In the similar way as the 4.31) was obtained, we have

$$
\sup _{0 \leq u \leq T} \mathbb{E}\left|e_{\Delta}^{\star}\left(u \wedge \theta_{\Delta, R}\right)\right|^{q} \leq \frac{1}{(1-v)^{q}} \sup _{0 \leq u \leq T} \mathbb{E}\left|e_{\Delta}\left(u \wedge \theta_{\Delta, R}\right)\right|^{q} .
$$

By the stochastic inequality and Assumption 2.1, we have that for any $t \in[0, T]$,

$$
\begin{aligned}
\mathbb{E}\left|e_{\Delta}\left(t \wedge \theta_{R}\right)\right|^{q} \leq & C \mathbb{E} \int_{0}^{t \wedge \theta_{R}}\left|f(x(s), x(s-\tau))-f_{\Delta}\left(\bar{Y}_{\Delta}(s), \bar{Y}_{\Delta}(s-\tau)\right)\right|^{q} d s \\
& +C \mathbb{E} \int_{0}^{t \wedge \theta_{R}}\left|g(x(s), x(s-\tau))-g_{\Delta}\left(\bar{Y}_{\Delta}(s), \bar{Y}_{\Delta}(s-\tau)\right)\right|^{q} d s \\
\leq & C_{R} \mathbb{E} \int_{0}^{t \wedge \theta_{R}}\left[\left|x(s)-Y_{\Delta}(s)\right|^{q}+\left|x(s-\tau)-Y_{\Delta}(s-\tau)\right|^{q}\right] d s \\
& +C \mathbb{E} \int_{0}^{t \wedge \theta_{R}}\left|f\left(Y_{\Delta}(s), Y_{\Delta}(s-\tau)\right)-f\left(\bar{Y}_{\Delta}(s), \bar{Y}_{\Delta}(s-\tau)\right)\right|^{q} d s \\
& +C \mathbb{E} \int_{0}^{t \wedge \theta_{R}}\left|f\left(\bar{Y}_{\Delta}(s), \bar{Y}_{\Delta}(s-\tau)\right)-f_{\Delta}\left(\bar{Y}_{\Delta}(s), \bar{Y}_{\Delta}(s-\tau)\right)\right|^{q} d s \\
& +C \mathbb{E} \int_{0}^{t \wedge \theta_{R}}\left|g\left(Y_{\Delta}(s), Y_{\Delta}(s-\tau)\right)-g\left(\bar{Y}_{\Delta}(s), \bar{Y}_{\Delta}(s-\tau)\right)\right|^{q} d s \\
& +C \mathbb{E} \int_{0}^{t \wedge \theta_{R}}\left|g\left(\bar{Y}_{\Delta}(s), \bar{Y}_{\Delta}(s-\tau)\right)-g_{\Delta}\left(\bar{Y}_{\Delta}(s), \bar{Y}_{\Delta}(s-\tau)\right)\right|^{q} d s \\
= & \sum_{j=1}^{5} \Pi_{j}(t) .
\end{aligned}
$$

By $[2.2)$, we get

$$
\Pi_{1}(t) \leq C_{R} \int_{0}^{T} \sup _{0 \leq u \leq s} \mathbb{E}\left|e_{\Delta}^{\star}\left(u \wedge \theta_{\Delta, R}\right)\right|^{q} d s+C_{R} \Delta^{\varrho q} .
$$

By Assumption 2.1 2.2) and (4.58), we have

$$
\Pi_{2}(t)+\Pi_{4}(t) \leq C_{R} \int_{0}^{T} \mathbb{E}\left|Y_{\Delta}(s)-\bar{Y}_{\Delta}(s)\right|^{q} d s+C_{R} \int_{0}^{T}|\xi(s)-\xi(\kappa(s))|^{q} d s \leq C_{R} \Delta^{(0.5 \wedge \varrho) q} .
$$


According to P1, we obtain

$$
\begin{aligned}
\Pi_{3}(t) & \leq C \int_{0}^{T} \mathbb{E}\left|f\left(\bar{Y}_{\Delta}\left(s \wedge \theta_{\Delta, R}\right), \bar{Y}_{\Delta}\left(s \wedge \theta_{\Delta, R}-\tau\right)\right)-f_{\Delta}\left(\bar{Y}_{\Delta}\left(s \wedge \theta_{\Delta, R}\right), \bar{Y}_{\Delta}\left(s \wedge \theta_{\Delta, R}-\tau\right)\right)\right|^{q} d s \\
& \leq C N_{R}^{q} \Delta^{\alpha q}=C_{R} \Delta^{\alpha q} .
\end{aligned}
$$

Similarly, we can show

$$
\Pi_{5}(t) \leq C_{R} \Delta^{\alpha q}
$$

From (4.61)-(4.64), we derive from 4.60) that

$$
\sup _{0 \leq u \leq T} \mathbb{E}\left|e_{\Delta}\left(u \wedge \theta_{\Delta, R}\right)\right|^{q} \leq C_{R} \int_{0}^{T} \sup _{0 \leq u \leq s} \mathbb{E}\left|e_{\Delta}^{\star}\left(u \wedge \theta_{\Delta, R}\right)\right|^{q} d s+C_{R} \Delta^{(\alpha \wedge \varrho) q} .
$$

In the light of 4.59) and 4.65, we have

$$
\sup _{0 \leq u \leq T} \mathbb{E}\left|e_{\Delta}^{\star}\left(u \wedge \theta_{\Delta, R}\right)\right|^{q} \leq C_{R} \int_{0}^{T} \sup _{0 \leq u \leq s} \mathbb{E}\left|e_{\Delta}^{\star}\left(u \wedge \theta_{\Delta, R}\right)\right|^{q} d s+C_{R} \Delta^{(\alpha \wedge \varrho) q} .
$$

Now, using the Gronwall inequality gives the assertion (4.57). Inserting (4.57) into 4.56) gives

$$
\mathbb{E}\left|e_{\Delta}^{\star}(T)\right|^{q} \leq \frac{C q \eta}{p_{0}}+\frac{C\left(p_{0}-q\right)}{p_{0} R^{2} \eta^{q /\left(p_{0}-q\right)}}+C_{R} \Delta^{(\alpha \wedge \varrho) q} .
$$

Then for any $\varepsilon>0$ we can choose $\eta$ such that

$$
\frac{C q \eta}{p_{0}}<\frac{\varepsilon}{3}
$$

and then take $R$ such that

$$
\frac{C\left(p_{0}-q\right)}{p_{0} R^{2} \eta^{q /\left(p_{0}-q\right)}}<\frac{\varepsilon}{3},
$$

finally for such $R$ choose $\Delta$ sufficiently small for

$$
C_{R} \Delta^{(\alpha \bumpeq \varrho) q}<\frac{\varepsilon}{3}
$$

so that, in 4.67,

$$
\mathbb{E}\left|e_{\Delta}^{\star}(T)\right|^{q}<\varepsilon
$$

as required.

\section{Mean-square stability}

Let us concentrate on the mean-square stability of the tamed EM scheme for NSDDE (2.1) in this section. We assume that $f$ and $g$ can be decomposed as $f(x, y)=F_{1}(x, y)+F(x, y)$ and $g(x, y)=G_{1}(x, y)+G(x, y)$, where $F_{1}, F: \mathbb{R}^{d} \times \mathbb{R}^{d} \rightarrow \mathbb{R}^{d}$ and $G_{1}, G: \mathbb{R}^{d} \times \mathbb{R}^{d} \rightarrow \mathbb{R}^{d \times n}$. Moreover,

$$
F_{1}(0,0)=F(0,0)=G_{1}(0,0)=G(0,0)=0,
$$

the coefficients $F_{1}, F, G_{1}, G$ satisfy the following conditions. 
Assumption 5.1. For any $R>0$, there exists constants $\hat{L}$ and $\tilde{L}_{R}$ depending on $R$ such that

$$
\left|F_{1}(x, y)-F_{1}(\bar{x}, \bar{y})\right| \vee\left|G_{1}(x, y)-G_{1}(\bar{x}, \bar{y})\right| \leq \hat{L}(|x-\bar{x}|+|y-\bar{y}|),
$$

for any $x, \bar{x}, y, \bar{y} \in \mathbb{R}^{d}$ and

$$
|F(x, y)-F(\bar{x}, \bar{y})| \vee|G(x, y)-G(\bar{x}, \bar{y})| \leq \tilde{L}_{R}(|x-\bar{x}|+|y-\bar{y}|),
$$

for any $x, \bar{x}, y, \bar{y} \in \mathbb{R}^{d}$ with $|x| \vee|y| \vee|\bar{x}| \vee|\bar{y}| \leq R$.

Assumption 5.2. There exist nonnegative constants $\vartheta, \lambda_{1}, \lambda_{2}, \lambda_{3}$ and $\lambda_{4}$ satisfying $\lambda_{1}>\lambda_{2}+$ $\lambda_{3}+\lambda_{4}$ such that

$$
\begin{aligned}
& 2\left\langle x-D(y), F_{1}(x, y)\right\rangle+(1+\vartheta)\left|G_{1}(x, y)\right|^{2} \leq-\lambda_{1}|x|^{2}+\lambda_{2}|y|^{2}, \\
& 2\langle x-D(y), F(x, y)\rangle+\left(1+\vartheta^{-1}\right)|G(x, y)|^{2} \leq \lambda_{3}|x|^{2}+\lambda_{4}|y|^{2},
\end{aligned}
$$

for any $x, y \in \mathbb{R}^{d}$.

When $\vartheta=0$, we set $\vartheta^{-1}|G(x, y)|^{2}=0$, when $\vartheta=\infty$, we set $\vartheta\left|G_{1}(x, y)\right|^{2}=0$. Moreover, we see from Assumption 5.2 that

$$
2\langle x-D(y), f(x, y)\rangle+|g(x, y)|^{2} \leq-\left(\lambda_{1}-\lambda_{3}\right)|x|^{2}+\left(\lambda_{2}+\lambda_{4}\right)|y|^{2}, \forall x, y \in \mathbb{R}^{d} .
$$

Thus, the solution to NSDDEs (2.1) is stable exponentially in mean-square sense, see e.g., Zong and $\mathrm{Wu}$ [10, Theorem 3.1]. We state this result as a lemma.

Lemma 5.3. Suppose that Assumptions 5.1 and 5.2 hold. Then for any initial data $\xi \in C\left([-\tau, 0] ; \mathbb{R}^{d}\right)$, the solution $x(t ; \xi)$ to the NSDDE (2.1) has the property that

$$
\limsup _{t \rightarrow \infty} \frac{\log \mathbb{E}|x(t ; \xi)|^{2}}{t} \leq-\left(\gamma^{\star} \wedge \frac{2}{\tau} \log \frac{1}{v}\right)
$$

where $\gamma^{\star}$ is the unique root of the following equation

$$
\gamma^{\star}(1+v)-\left(\lambda_{1}-\lambda_{3}\right)+e^{\gamma^{\star} \tau}\left(\gamma^{\star} v(v+1)+\lambda_{2}+\lambda_{4}\right)=0 .
$$

The following lemma shows that the partially tamed coefficients $f_{\Delta}$ and $g_{\Delta}$ conserve the stable condition 5.3

Lemma 5.4. Suppose that Assumptions 5.1 and 5.2 hold and $\alpha \in(0,1 / 3]$ is arbitrary. Define the following partially modified coefficients $f_{\Delta}$ and $g_{\Delta}$ by

$$
f_{\Delta}(x, y)=F_{1}(x, y)+F_{\Delta}(x, y) \quad \text { and } \quad g_{\Delta}(x, y)=G_{1}(x, y)+G_{\Delta}(x, y),
$$

where

$$
F_{\Delta}(x, y):=\pi_{\Delta}(x, y) F(x, y) \quad \text { and } \quad G_{\Delta}(x, y):=\pi_{\Delta}(x, y) G(x, y)
$$

with

$$
\pi_{\Delta}(x, y)=\frac{1}{1+\Delta^{\alpha}\left(|F(x, y)|+|G(x, y)|^{2}\right)}, \forall x, y \in \mathbb{R}^{d}, \Delta \in(0,1] .
$$


Then

$$
2(x-D(y))^{T} f_{\Delta}(x, y)+\left|g_{\Delta}(x, y)\right|^{2} \leq-\left(\lambda_{1}-\lambda_{3}\right)|x|^{2}+\left(\lambda_{2}+\lambda_{4}\right)|y|^{2}, \forall x, y \in \mathbb{R}^{d},
$$

and

$$
\left|f_{\Delta}(x, y)\right|^{2} \Delta \leq \epsilon_{\Delta}\left(|x|^{2}+|y|^{2}\right), \forall x, y \in \mathbb{R}^{d},
$$

135 where $\epsilon_{\Delta}=4\left(\hat{L}+\tilde{L}_{1}\right)^{2} \Delta+4 \Delta^{1-2 \alpha}$.

Proof. Write $\pi_{\Delta}(x, y)=\pi_{\Delta}$ for short. Note that $0<\pi_{\Delta}<1$. By Assumptions 5.1 and 5.2 as well as (5.6), we have

$$
\begin{aligned}
2(x-D(y))^{T} F_{\Delta}(x, y)+\left(1+\vartheta^{-1}\right)\left|G_{\Delta}(x, y)\right|^{2} & =2(x-D(y))^{T} \pi_{\Delta} F(x, y)+\left(1+\vartheta^{-1}\right)\left|\pi_{\Delta} G(x, y)\right|^{2} \\
& \leq 2 \pi_{\Delta}(x-D(y))^{T} F(x, y)+\pi_{\Delta}\left(1+\vartheta^{-1}\right)|G(x, y)|^{2} \\
& \leq \pi_{\Delta}\left(\lambda_{3}|x|^{2}+\lambda_{4}|y|^{2}\right) \\
& \leq \lambda_{3}|x|^{2}+\lambda_{4}|y|^{2}, \forall x, y \in \mathbb{R}^{d}
\end{aligned}
$$

Consequently,

$$
\begin{aligned}
2(x-D(y))^{T} f_{\Delta}(x, y)+\left|g_{\Delta}(x, y)\right|^{2} \leq & 2(x-D(y))^{T} F_{1}(x, y)+(1+\vartheta)\left|G_{1}(x, y)\right|^{2} \\
& +2(x-D(y))^{2} F_{\Delta}(x, y)+\left(1+\vartheta^{-1}\right)\left|G_{\Delta}(x, y)\right|^{2} \\
\leq & -\left(\lambda_{1}-\lambda_{3}\right)|x|^{2}+\left(\lambda_{2}+\lambda_{4}\right)|y|^{2}, \forall x, y \in \mathbb{R}^{d} .
\end{aligned}
$$

Now let us estimate 5.8. By Assumption 5.1 and condition (5.1), we have

$$
\left|F_{1}(x, y)\right| \leq \hat{L}(|x|+|y|), \forall x, y \in \mathbb{R}^{d} .
$$

For any $x, y \in \mathbb{R}^{d}$ with $|x| \vee|y| \leq 1$, by Assumption 5.1 and condition 5.1, we have

$$
\left|F_{\Delta}(x, y)\right|=\left|\pi_{\Delta} F(x, y)\right| \leq|F(x, y)| \leq \tilde{L}_{1}(|x|+|y|) .
$$

While for any $x, y \in \mathbb{R}^{d}$ with $|x| \vee|y|>1$, by 5.6, we have

$$
\left|F_{\Delta}(x, y)\right|=\frac{|F(x, y)|}{1+\Delta^{\alpha}\left(|F(x, y)|+|G(x, y)|^{2}\right)} \leq \Delta^{-\alpha} \leq \Delta^{-\alpha}(|x|+|y|) .
$$

Thus,

$$
\left|F_{\Delta}(x, y)\right| \leq\left(\tilde{L}_{1}+\Delta^{-\alpha}\right)(|x|+|y|), \forall x, y \in \mathbb{R}^{d} .
$$

Consequently, by 5.11 and 5.12, we obtain

$$
\begin{aligned}
\left|f_{\Delta}(x, y)\right|^{2} \Delta & \leq\left(\hat{L}+\tilde{L}_{1}+\Delta^{-\alpha}\right)^{2}(|x|+|y|)^{2} \Delta \\
& \leq 4\left(\hat{L}+\tilde{L}_{1}\right)^{2} \Delta+4 \Delta^{1-2 \alpha}\left(|x|^{2}+|y|^{2}\right), \forall x, y \in \mathbb{R}^{d} .
\end{aligned}
$$

Thus, the proof is complete.

The following theorem shows that the tamed EM solution can share the mean-square stability of the exact solution. 
Theorem 5.5. Suppose that Assumptions 5.1 and 5.2 hold and $\alpha \in(0,1 / 3]$ is arbitrary. Choose $\Delta^{\star} \in(0,1]$ such that $\epsilon_{\Delta^{\star}} \leq\left(\lambda_{1}-\lambda_{2}-\lambda_{3}-\lambda_{4}\right) / 2$, where $\epsilon_{\Delta}$ is defined in $(5.8)$. Then for any $\Delta \in\left(0, \Delta^{\star}\right]$ and any initial data $\xi \in C\left([-\tau, 0] ; \mathbb{R}^{d}\right)$, the tamed EM approximation $y_{\Delta}^{k}$ defined by (3.1) with modified coefficients (5.6) has the property that

$$
\limsup _{k \rightarrow \infty} \frac{\log \left(\mathbb{E}\left|y_{\Delta}^{k}\right|^{2}\right)}{k \Delta} \leq-\left(\gamma_{\Delta}^{\star} \wedge \frac{2}{\tau} \log \frac{1}{v}\right)
$$

where $\gamma_{\Delta}^{\star}$ is the unique root of the following equation

$$
\frac{1-e^{-\gamma_{\Delta}^{\star} \Delta}}{\Delta}(1+v)-\left(\lambda_{1}-\lambda_{3}-\epsilon_{\Delta}\right)+e^{\gamma_{\Delta}^{\star} \tau}\left(\frac{1-e^{-\gamma_{\Delta}^{\star} \Delta}}{\Delta} v(1+v)+\left(\lambda_{2}+\lambda_{4}+\epsilon_{\Delta}\right)\right)=0 .
$$

Moreover,

$$
\lim _{\Delta \rightarrow 0} \gamma_{\Delta}^{\star}=\gamma^{\star}
$$

Proof. Let Assumptions 5.1 and 5.2 hold. Consider the tamed EM scheme 3.1 with $f_{\Delta}$ and $g_{\Delta}$ given by (5.6. Then

$$
\begin{aligned}
\left|y_{\Delta}^{k+1}-D\left(y_{\Delta}^{k+1-m}\right)\right|^{2}= & \left|y_{\Delta}^{k}-D\left(y_{\Delta}^{k-m}\right)\right|^{2}+2\left(y_{\Delta}^{k}-D\left(y_{\Delta}^{k-m}\right)\right)^{T} f_{\Delta}\left(y_{\Delta}^{k}, y_{\Delta}^{k-m}\right) \Delta \\
& +\left|g_{\Delta}\left(y_{\Delta}^{k}, y_{\Delta}^{k-m}\right)\right|^{2} \Delta+\left|f_{\Delta}\left(y_{\Delta}^{k}, y_{\Delta}^{k-m}\right)\right|^{2} \Delta^{2}+M_{k}, \quad k=0,1,2, \cdots,
\end{aligned}
$$

where

$$
\begin{aligned}
M_{k}= & 2\left(y_{\Delta}^{k}-D\left(y_{\Delta}^{k-m}\right)\right)^{T} g_{\Delta}\left(y_{\Delta}^{k}, y_{\Delta}^{k-m}\right) \Delta B_{k}+2\left(f_{\Delta}\left(y_{\Delta}^{k}, y_{\Delta}^{k-m}\right)\right)^{T}\left[g_{\Delta}\left(y_{\Delta}^{k}, y_{\Delta}^{k-m}\right) \Delta B_{k}\right] \Delta \\
& +\left|g_{\Delta}\left(y_{\Delta}^{k}, y_{\Delta}^{k-m}\right) \Delta B_{k}\right|^{2}-\left|g_{\Delta}\left(y_{\Delta}^{k}, y_{\Delta}^{k-m}\right)\right|^{2} \Delta .
\end{aligned}
$$

Obviously, $\mathbb{E} M_{k}=0$. Denote $z_{\Delta}^{k}:=y_{\Delta}^{k}-D\left(y_{\Delta}^{k-m}\right)$. Now choose $\Delta^{\star} \in(0,1]$ such that $\epsilon_{\Delta^{\star}} \leq$ $\left(\lambda_{1}-\lambda_{2}-\lambda_{3}-\lambda_{4}\right) / 2$, where $\epsilon_{\Delta^{\star}}$ is defined in Lemma 5.4. Then for any $\Delta \in\left(0, \Delta^{\star}\right]$, taking expectations on both sides of 5.16 and applying Lemma 5.4, we have

$$
\begin{aligned}
\mathbb{E}\left|z_{\Delta}^{k+1}\right|^{2} & \leq \mathbb{E}\left|z_{\Delta}^{k}\right|^{2}-\left(\lambda_{1}-\lambda_{3}\right) \mathbb{E}\left|y_{\Delta}^{k}\right|^{2} \Delta+\left(\lambda_{2}+\lambda_{4}\right) \mathbb{E}\left|y_{\Delta}^{k-m}\right|^{2} \Delta+\epsilon_{\Delta}\left(\mathbb{E}\left|y_{\Delta}^{k}\right|^{2}+\mathbb{E}\left|y_{\Delta}^{k-m}\right|^{2}\right) \Delta \\
& =\mathbb{E}\left|z_{\Delta}^{k}\right|^{2}-\left(\lambda_{1}-\lambda_{3}-\epsilon_{\Delta}\right) \mathbb{E}\left|y_{\Delta}^{k}\right|^{2} \Delta+\left(\lambda_{2}+\lambda_{4}+\epsilon_{\Delta}\right) \mathbb{E}\left|y_{\Delta}^{k-m}\right|^{2} \Delta .
\end{aligned}
$$

For any $r>1$, we have

$$
\begin{aligned}
r^{(k+1) \Delta} \mathbb{E}\left|z_{\Delta}^{k+1}\right|^{2}-r^{k \Delta} \mathbb{E}\left|z_{\Delta}^{k}\right|^{2} \leq & -\left(\lambda_{1}-\lambda_{3}-\epsilon_{\Delta}\right) r^{(k+1) \Delta} \mathbb{E}\left|y_{\Delta}^{k}\right|^{2} \Delta+\left(\lambda_{2}+\lambda_{4}+\epsilon_{\Delta}\right) r^{(k+1) \Delta} \mathbb{E}\left|y_{\Delta}^{k-m}\right|^{2} \Delta \\
& +\left(r^{(k+1) \Delta}-r^{k \Delta}\right) \mathbb{E}\left|z_{\Delta}^{k}\right|^{2}
\end{aligned}
$$

By the elementary inequality, we have

$$
|x-D(y)|^{2} \leq(1+v)|x|^{2}+\left(1+v^{-1}\right) v^{2}|y|^{2}=(1+v)|x|^{2}+\left(v^{2}+v\right)|y|^{2}, \quad \forall x, y \in \mathbb{R}^{d} .
$$


Then we see from (5.18) that

$$
\begin{aligned}
r^{k \Delta} \mathbb{E}\left|z_{\Delta}^{k}\right|^{2} \leq & \mathbb{E}\left|z_{\Delta}^{0}\right|^{2}-\left(\lambda_{1}-\lambda_{3}-\epsilon_{\Delta}\right) \sum_{j=0}^{k-1} r^{(j+1) \Delta} \mathbb{E}\left|y_{\Delta}^{j}\right|^{2} \Delta+\left(\lambda_{2}+\lambda_{4}+\epsilon_{\Delta}\right) \sum_{j=0}^{k-1} r^{(j+1) \Delta} \mathbb{E}\left|y_{\Delta}^{j-m}\right|^{2} \Delta \\
& +\sum_{j=0}^{k-1}\left(r^{(j+1) \Delta}-r^{j \Delta}\right)\left((1+v) \mathbb{E}\left|y_{\Delta}^{j}\right|^{2}+\left(v^{2}+v\right) \mathbb{E}\left|y_{\Delta}^{j-m}\right|^{2}\right) \\
= & |\xi(0)-D(\xi(-\tau))|^{2}+\left[-\left(\lambda_{1}-\lambda_{3}-\epsilon_{\Delta}\right) \Delta+\left(1-r^{-\Delta}\right)(1+v)\right] \sum_{j=0}^{k-1} r^{(j+1) \Delta} \mathbb{E}\left|y_{\Delta}^{j}\right|^{2} \\
& +\left[\left(\lambda_{2}+\lambda_{4}+\epsilon_{\Delta}\right) \Delta+\left(1-r^{-\Delta}\right)\left(v^{2}+v\right)\right] \sum_{j=0}^{k-1} r^{(j+1) \Delta} \mathbb{E}\left|y_{\Delta}^{j-m}\right|^{2}, k=1,2, \cdots
\end{aligned}
$$

Note that

$$
\begin{aligned}
\sum_{j=0}^{k-1} r^{(j+1) \Delta} \mathbb{E}\left|y_{\Delta}^{j-m}\right|^{2} & =\sum_{j=-m}^{-1} r^{(j+1+m) \Delta} \mathbb{E}\left|y_{\Delta}^{j}\right|^{2}+\sum_{j=0}^{k-m-1} r^{(j+1+m) \Delta} \mathbb{E}\left|y_{\Delta}^{j}\right|^{2} \\
& \leq \frac{r^{\tau}}{1-r^{-\Delta}}\|\xi\|^{2}+r^{\tau} \sum_{j=0}^{k-1} r^{(j+1) \Delta} \mathbb{E}\left|y_{\Delta}^{j}\right|^{2}, k=1,2, \cdots
\end{aligned}
$$

Substituting this into 5.20 , we obtain

$$
r^{k \Delta} \mathbb{E}\left|z_{\Delta}^{k}\right|^{2} \leq H_{\Delta}(r)-\bar{H}_{\Delta}(r) \sum_{j=0}^{k-1} r^{(j+1) \Delta} \mathbb{E}\left|y_{\Delta}^{j}\right|^{2} \Delta, k=1,2, \cdots,
$$

where

$$
\begin{gathered}
H_{\Delta}(r):=\left[\frac{r^{\tau}}{1-r^{-\Delta}}\left[\left(\lambda_{2}+\lambda_{4}+\epsilon_{\Delta}\right) \Delta+\left(1-r^{-\Delta}\right)\left(v^{2}+v\right)\right]+(1+v)^{2}\right]\|\xi\|^{2}, \\
\bar{H}_{\Delta}(r):=\left(\lambda_{1}-\lambda_{3}-\epsilon_{\Delta}\right)-\frac{1-r^{-\Delta}}{\Delta}(1+v)-\left[\left(\lambda_{2}+\lambda_{4}+\epsilon_{\Delta}\right)+\frac{1-r^{-\Delta}}{\Delta}\left(v^{2}+v\right)\right] r^{\tau} .
\end{gathered}
$$

For any $\Delta \in\left(0, \Delta^{\star}\right]$, we have

$$
\bar{H}_{\Delta}(1)=\lambda_{1}-\lambda_{2}-\lambda_{3}-\lambda_{4}-2 \epsilon_{\Delta}>0,
$$

and

$$
\bar{H}_{\Delta}(\bar{r})<0, \text { with } \bar{r}=\left(\frac{\lambda_{1}-\lambda_{3}-\epsilon_{\Delta}}{\lambda_{2}+\lambda_{4}+\epsilon_{\Delta}}\right)^{1 / \tau}>1,
$$

as well as

$$
\frac{d \bar{H}_{\Delta}(r)}{d r}<0 .
$$


From 5.25- 5.27 , there is a positive constant $r_{\Delta}^{\star} \in(1, \bar{r})$ such that $\bar{H}_{\Delta}\left(r_{\Delta}^{\star}\right)=0$. If $1<r<r_{\Delta}^{\star}$ and $1<r<v^{-\frac{2}{\tau}}$, which means $1-r^{\tau} v^{2}>0$, by the elementary inequality and the connection between $y_{\Delta}^{k}$ and $z_{\Delta}^{k}$, we see from (5.22) that

$$
\begin{aligned}
r^{k \Delta} \mathbb{E}\left|y_{\Delta}^{k}\right|^{2} & \leq r^{k \Delta}(1+\eta) \mathbb{E}\left|z_{\Delta}^{k}\right|^{2}+r^{k \Delta}\left(1+\eta^{-1}\right) v^{2} \mathbb{E}\left|y_{\Delta}^{k-m}\right|^{2} \\
& \leq(1+\eta) H_{\Delta}\left(r_{\Delta}^{\star}\right)+\left(r^{\tau}\left(1+\eta^{-1}\right) v^{2}\right) r^{(k-m) \Delta} \mathbb{E}\left|y_{\Delta}^{k-m}\right|^{2}, k=0,1,2, \cdots,
\end{aligned}
$$

where inequality $r^{k \Delta} \mathbb{E}\left|z_{\Delta}^{k}\right|^{2}<\left(r_{\Delta}^{\star}\right)^{k \Delta} \mathbb{E}\left|z_{\Delta}^{k}\right|^{2} \leq H_{\Delta}\left(r_{\Delta}^{\star}\right)$ has been used and $\eta$ is a positive constant to be determined. Denote $a_{k}:=r^{k \Delta} \mathbb{E}\left|y_{\Delta}^{k}\right|^{2}$, then $(5.28)$ becomes

$$
a_{k} \leq(1+\eta) H_{\Delta}\left(r_{\Delta}^{\star}\right)+\left(r^{\tau}\left(1+\eta^{-1}\right) v^{2}\right) a_{k-m}, k=0,1,2, \cdots .
$$

Hence,

$$
\begin{aligned}
& a_{i} \leq(1+\eta) H_{\Delta}\left(r_{\Delta}^{\star}\right)+\left(r^{\tau}\left(1+\eta^{-1}\right) v^{2}\right) \sup _{-m \leq i \leq k} a_{i}, 0 \leq i \leq k, \\
& a_{i} \leq\|\xi\|^{2}<H_{\Delta}\left(r_{\Delta}^{\star}\right),-m \leq i \leq 0 .
\end{aligned}
$$

Thus,

$$
\sup _{-m \leq i \leq k} a_{i} \leq(1+\eta) H_{\Delta}\left(r_{\Delta}^{\star}\right)+\left(r^{\tau}\left(1+\eta^{-1}\right) v^{2}\right) \sup _{-m \leq i \leq k} a_{i}, k=0,1,2, \cdots .
$$

Now, taking $\eta>\frac{r^{\tau} v^{2}}{1-r^{\tau} v^{2}}$, i.e., $r^{\tau}\left(1+\eta^{-1}\right) v^{2}<1$, we obtain from 5.30] that

$$
\sup _{-m \leq i \leq k} r^{i \Delta} \mathbb{E}\left|y_{\Delta}^{i}\right|^{2} \leq \frac{(1+\eta) H_{\Delta}\left(r_{\Delta}^{\star}\right)}{1-r^{\tau}\left(1+\eta^{-1}\right) v^{2}}<\infty, k=0,1,2, \cdots .
$$

Therefore,

$$
\limsup _{k \rightarrow \infty} \frac{\log \mathbb{E}\left|y_{\Delta}^{k}\right|^{2}}{k \Delta} \leq-\log r
$$

Similarly, if $1<r_{\Delta}^{\star} \leq r$ and $1<r<v^{-\frac{2}{\tau}}$, replacing $r$ by $r_{\Delta}^{\star}$ in the above procedure between (5.28) and 5.31, we also have

$$
\limsup _{k \rightarrow \infty} \frac{\log \mathbb{E}\left|y_{\Delta}^{k}\right|^{2}}{k \Delta} \leq-\log r_{\Delta}^{\star} .
$$

Combining (5.32) and (5.33) gives

$$
\limsup _{k \rightarrow \infty} \frac{\log \mathbb{E}\left|y_{\Delta}^{k}\right|^{2}}{k \Delta} \leq-\log \left(r_{\Delta}^{\star} \wedge r\right), \text { with } 1<r<v^{-\frac{2}{\tau}},
$$

where $r_{\Delta}^{\star}$ is the unique root of 5.24. Taking $r=\mathrm{e}^{\gamma} \rightarrow v^{-\frac{2}{\tau}}$ and $r_{\Delta}^{\star}=\mathrm{e}^{\gamma_{\Delta}^{\star}}$, then 5.34 becomes 140 5.13. Finally, notice that $\epsilon_{\Delta} \rightarrow 0$ and $\frac{1-\mathrm{e}^{-\gamma_{\Delta}^{\star} \Delta}}{\Delta} \rightarrow \gamma_{\Delta}^{\star}$ as $\Delta \rightarrow 0$. Comparing 5.5] with 5.14, we obtain the desired assertion (5.15). Thus, the proof is finished. 


\section{Numerical examples}

In this section, we carry out some numerical experiments to support the findings derived. In the following two examples, the diffusion coefficients of NSDDEs are superlinearly growing and therefore one can not apply the results of $\mathrm{Li}$ and $\mathrm{Cao}[\overline{3}]$, Ji and Yuan [5], Zong and $\mathrm{Wu}[10]$ and Tan [11].

Example 6.1. Consider the following one-dimensional NSDDE:

$$
\begin{aligned}
d\left[x(t)+\frac{1}{2} x(t-\tau)\right]= & {\left[6 x(t)+\frac{5}{2} x(t-\tau)-5\left(x(t)+\frac{1}{2} x(t-\tau)\right)\left|x(t)+\frac{1}{2} x(t-\tau)\right|\right] d t } \\
& +\left[x(t)+\sin x(t-\tau)+\left|x(t)+\frac{1}{2} x(t-\tau)\right|^{3 / 2}\right] d B(t), t \geq 0, \\
x(t)= & 1,-\tau \leq t \leq 0,
\end{aligned}
$$

where $B(t)$ is a scalar Wiener process and $\tau=1 / 8$. We first verify the Assumptions 2.1, 2.2 and 2.4 Obviously, Assumptions 2.1 is satisfied. For any $x, y \in \mathbb{R}$, set $f(x, y)=f_{1}(x, y)+f_{2}(x, y)$, $g(x, y)=g_{1}(x, y)+g_{2}(x, y)$, where $f_{1}(x, y)=a(x+0.5 y)-a(x+0.5 y)|x+0.5 y|, f_{2}(x, y)=x$, $g_{1}(x, y)=|x+0.5 y|^{3 / 2}, g_{2}(x, y)=x+\sin y$, and $a=5$. Donote $D(y)=-0.5 y, X:=x+0.5 y=$ $x-D(y), Y:=\bar{x}+0.5 \bar{y}=\bar{x}-D(\bar{y}), F(X):=a X(1-|X|), G(X):=|X|^{3 / 2}$. Then $f_{1}(x, y)=F(X)$, $g_{1}(x, y)=G(X), f_{1}(\bar{x}, \bar{y})=F(Y), g_{1}(\bar{x}, \bar{y})=G(Y)$. If $4 \leq p_{0} \leq a+1=6$, then

$$
\begin{aligned}
& 2\langle x-D(y), f(x, y)\rangle+\left(p_{0}-1\right)|g(x, y)|^{2} \\
& =2(x+0.5 y)\left(6 x+\frac{5}{2} y-a(x+0.5 y)|x+0.5 y|\right)+\left(p_{0}-1\right)\left(x+\sin y+|x+0.5 y|^{3 / 2}\right)^{2} \\
& \leq 2(x+0.5 y)\left(6 x+\frac{5}{2} y\right)+2\left(p_{0}-1\right)(x+\sin y)^{2}+2\left(p_{0}-1-a\right)|x+0.5 y|^{3} \\
& \leq 2(x+0.5 y)\left(6 x+\frac{5}{2} y\right)+2\left(p_{0}-1\right)(x+\sin y)^{2} \\
& \leq K_{2}\left(1+|x|^{2}+|y|^{2}\right),
\end{aligned}
$$

which means that Assumption 2.2 is satisfied. Moreover, we can derive that

$$
\begin{aligned}
& \left\langle x-D(y)-\bar{x}+D(\bar{y}), f_{1}(x, y)-f_{1}(\bar{x}, \bar{y})\right\rangle=\langle X-Y, a(X-Y)-a(X|X|-Y|Y|)\rangle \\
& =a|X-Y|^{2}-a(X-Y)(X|X|-Y|Y|) \leq a|X-Y|^{2}-a(|X|+|Y|)(|X|-|Y|)^{2},
\end{aligned}
$$

where we have used the following estimates

$$
-\langle X-Y, X|X|-Y|Y|\rangle \leq-(|X|+|Y|)(|X|-|Y|)^{2}, \forall X, Y \in \mathbb{R}^{d},
$$

and

$$
\left|g_{1}(x, y)-g_{1}(\bar{x}, \bar{y})\right|^{2}=|G(X)-G(Y)|^{2}=\left(|X|^{3 / 2}-|Y|^{3 / 2}\right)^{2} \leq 2(|X|+|Y|)(|X|-|Y|)^{2},
$$

see [21] Appendix, p.2104]. If $p_{1} \leq a / 2+1=3.5$, then we conclude from 6.3] and 6.4] that

$$
\begin{aligned}
& 2\left\langle x-D(y)-\bar{x}+D(\bar{y}), f_{1}(x, y)-f_{1}(\bar{x}, \bar{y})\right\rangle+2\left(p_{1}-1\right)\left|g_{1}(x, y)-g_{1}(\bar{x}, \bar{y})\right|^{2} \\
& \leq 2 a|X-Y|^{2}-2 a(|X|+|Y|)(|X|-|Y|)^{2}+4\left(p_{1}-1\right)(|X|+|Y|)(|X|-|Y|)^{2} \\
& =2 a|X-Y|^{2}+\left[4\left(p_{1}-1\right)-2 a\right](|X|+|Y|)(|X|-|Y|)^{2} \\
& \leq 2 a|X-Y|^{2}=2 a|x-\bar{x}-D(y)+D(\bar{y})|^{2} \leq 4 a\left(|x-\bar{x}|^{2}+|y-\bar{y}|^{2}\right) .
\end{aligned}
$$


Table 1: $\epsilon_{\Delta}$ and $\gamma_{\Delta}^{\star}$ with different step sizes for solving 5.14 in example 6.2.:

$\lambda_{1}=5 / 2, \lambda_{2}=13 / 8, \lambda_{3}=0, \lambda_{4}=1 / 4, \hat{L}=2, \tilde{L}_{1}=3, \tau=1, v=1 / 2, \alpha=1 / 4, \Delta^{\star}=0.0015$, $\frac{2}{\tau} \log \frac{1}{v}=1.3863, \gamma^{\star}=0.1427$

\begin{tabular}{ccccccc}
\hline$\Delta$ & $10^{-3}$ & $10^{-4}$ & $10^{-5}$ & $10^{-6}$ & $10^{-7}$ & $10^{-8}$ \\
\hline$\epsilon_{\Delta}$ & 0.2265 & 0.0500 & 0.0136 & 0.0041 & 0.0013 & 0.0004 \\
$\gamma_{\Delta}^{\star}$ & 0.0389 & 0.1196 & 0.1364 & 0.1408 & 0.1421 & 0.1425 \\
\hline
\end{tabular}

Thus,

$$
\begin{aligned}
& 2\langle x-D(y)-\bar{x}+D(\bar{y}), f(x, y)-f(\bar{x}, \bar{y})\rangle+\left(p_{1}-1\right)|g(x, y)-g(\bar{x}, \bar{y})|^{2} \\
\leq & 2\left\langle x-D(y)-\bar{x}+D(\bar{y}), f_{1}(x, y)-f_{1}(\bar{x}, \bar{y})\right\rangle+2\left(p_{1}-1\right)\left|g_{1}(x, y)-g_{1}(\bar{x}, \bar{y})\right|^{2} \\
& +2\left\langle x-D(y)-\bar{x}+D(\bar{y}), f_{2}(x, y)-f_{2}(\bar{x}, \bar{y})\right\rangle+2\left(p_{1}-1\right)\left|g_{2}(x, y)-g_{2}(\bar{x}, \bar{y})\right|^{2} \\
\leq & 4 a\left(|x-\bar{x}|^{2}+|y-\bar{y}|^{2}\right)+2\langle x+0.5 y-\bar{x}-0.5 \bar{y}, x-\bar{x}\rangle+2\left(p_{1}-1\right)|x-\bar{x}+\sin y-\sin \bar{y}|^{2} \\
\leq & 4 a\left(|x-\bar{x}|^{2}+|y-\bar{y}|^{2}\right)+2|x-\bar{x}|^{2}+|y-\bar{y}||x-\bar{x}|+4\left(p_{1}-1\right)\left(|x-\bar{x}|^{2}+|y-\bar{y}|^{2}\right) \\
\leq & 4\left(a+p_{1}\right)\left(|x-\bar{x}|^{2}+|y-\bar{y}|^{2}\right)
\end{aligned}
$$

which impies that the global monotonicity condition is satisfied. In addition,

$$
\begin{aligned}
\left|f_{1}(x, y)-f_{1}(\bar{x}, \bar{y})\right| & =a|(X-Y)-(X|X|-Y|Y|)| \\
& \leq a(1+|X|+|Y|)|X-Y| \\
& \leq a(1+|x|+|y|+|\bar{x}|+|\bar{y}|)(|x-\bar{x}|+|y-\bar{y}|) .
\end{aligned}
$$

Thus, we derive from the definitions of $f$ and $g$ that polynomial growth condition is satisfied. Taking $l=1, p_{0}=6, p_{1}=3.5, p=2$ and $\varrho=0.5$, we conclude from Theorem 4.1 that the tamed $E M$ solution $Y_{\Delta}(t)$ with modified coefficients Type II is convergent to the exact solution $x(t)$ with order one half in the sense of mean-square. Now define the root of mean-square error

$$
\hat{e}_{\Delta}(T):=\left(\mathbb{E}\left|x(T)-Y_{\Delta}(T)\right|^{2}\right)^{1 / 2} .
$$

Set $\alpha=1 / 2$, we apply the tamed EM scheme (3.1) with modified coefficients Type II to approximate the exact solution $x(t)$ of NSDDE 6.1]. Tamed EM solution $Y_{\Delta}(t)$ with step size $\Delta=2^{-14}$ is taken as the replacement of the exact solution $x(t)$. Fig.l( $(a)$ shows the root of mean-square errors $150 \hat{e}_{\Delta}(T)$ between the exact solution $x(T)$ and the tamed EM solution $Y_{\Delta}(T)$ with different step sizes $2^{-6}, 2^{-7}, \cdots 2^{-11}$ at time $T=1$ for 500 simulations. A least square fit of errors $\hat{e}_{\Delta}$ produces the strong convergence order 0.5431 and is thus close to the theoretical value 0.5 .

Example 6.2. Consider the following one-dimensional NSDDE:

$$
\begin{aligned}
d\left[x(t)-\frac{1}{2} \sin x(t-\tau)\right] & =\left[-2 x(t)-x^{3}(t)+\frac{1}{2} \sin x(t-\tau)\right] d t+\left[\frac{1}{2} x^{2}(t)+\frac{1}{4} x(t-\tau)\right] d B(t), t \geq 0, \\
x(t) & =2,-\tau \leq t \leq 0
\end{aligned}
$$




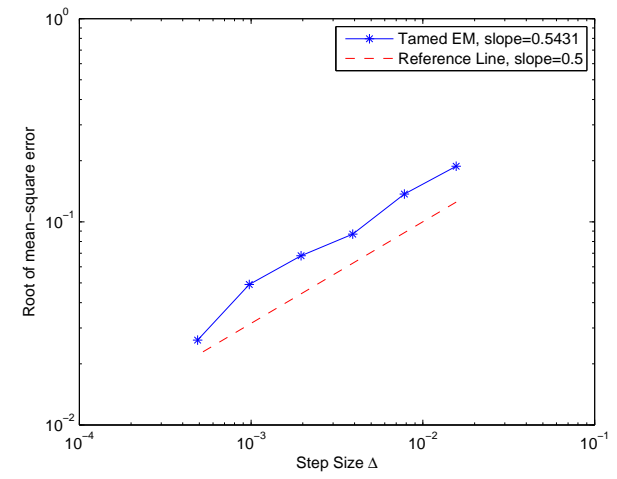

(a) Root of mean-square errors $\hat{e}_{\Delta}(T)$ for 6.1

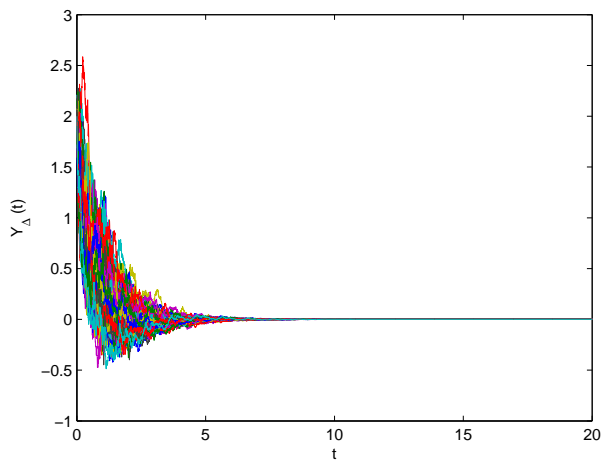

(b) 200 sample paths of $Y_{\Delta}(t)$ for 6.8

Fig. 1. Numerical simulations for 6.1 and 6.8

where $B(t)$ is a scalar Wiener process and $\tau=1$. Set $D(y)=\frac{1}{2} \sin y$ with $v=1 / 2, f(x, y)=$ $F_{1}(x, y)+F(x, y), g(x, y)=G_{1}(x, y)+G(x, y)$, where $F_{1}(x, y)=-2 x+\frac{1}{2} \sin y, G_{1}(x, y)=\frac{1}{4} y$, $F(x, y)=-x^{3}$ and $G(x, y)=\frac{1}{2} x^{2}$. We compute

$$
\left|F_{1}(x, y)-F_{1}(\bar{x}, \bar{y})\right| \vee\left|G_{1}(x, y)-G_{1}(\bar{x}, \bar{y})\right| \leq 2(|x-\bar{x}|+|y-\bar{y}|),
$$

for any $x, \bar{x}, y, \bar{y} \in \mathbb{R}$ and

$$
|F(x, y)-F(\bar{x}, \bar{y})| \vee|G(x, y)-G(\bar{x}, \bar{y})| \leq\left(3 R^{2} \vee R\right)(|x-\bar{x}|+|y-\bar{y}|),
$$

for any $x, \bar{x}, y, \bar{y} \in \mathbb{R}$ with $|x| \vee|y| \vee|\bar{x}| \vee|\bar{y}| \leq R$. Thus, Assumption 5.1 is satisfied. By the elementary inequality, we have the following estimates

$$
\begin{aligned}
& 2\left\langle x-D(y), F_{1}(x, y)\right\rangle+2\left|G_{1}(x, y)\right|^{2}=\left\langle 2 x-\sin y,-2 x+\frac{1}{2} \sin y\right\rangle+2\left(\frac{1}{4} y\right)^{2} \\
& =-4 x^{2}+3 x \sin y-\frac{1}{2} \sin ^{2} y+\frac{1}{8} y^{2} \leq-4 x^{2}+\frac{3}{2}\left(x^{2}+y^{2}\right)+\frac{1}{8} y^{2}=-\frac{5}{2} x^{2}+\frac{13}{8} y^{2},
\end{aligned}
$$

and

$$
\begin{aligned}
& 2\langle x-D(y), F(x, y)\rangle+2|G(x, y)|^{2}=\left\langle 2 x-\sin y,-x^{3}\right\rangle+2\left(\frac{1}{2} x^{2}\right)^{2} \\
& =-2 x^{4}+x^{3} \sin y+\frac{1}{2} x^{4}=-\frac{3}{2} x^{4}+x^{3} \sin y \leq-\frac{3}{2} x^{4}+\frac{3}{4} x^{4}+\frac{1}{4} \sin ^{4} y \\
& \leq-\frac{3}{2} x^{4}+\frac{3}{4} x^{4}+\frac{1}{4} y^{2} \leq \frac{1}{4} y^{2},
\end{aligned}
$$

where the Young inequality and the inequality that $\sin ^{2} y \leq y^{2}$ have been used. Thus Assumptions 5.2 is satisfied with $\lambda_{1}=\frac{5}{2}, \lambda_{2}=\frac{13}{8}, \lambda_{3}=0, \lambda_{4}=\frac{1}{4}$ and $\vartheta=1$. From 6.9] and 6.10, we have 
gives $\Delta^{\star}=0.0015$. Computational results such as $\epsilon_{\Delta}$ and $\gamma_{\Delta}^{\star}$ as well as $\gamma^{\star}$ are shown in Table 1 . According to Lemma 5.3, the exact solution $x(t)$ of NSDDE (6.8) is mean-square exponentially stable with exponent $-\left(\gamma^{\star} \wedge \frac{2}{\tau} \log \frac{1}{\gamma}\right)$. On the other hand, based on Theorem 5.5 the tamed EM solution $Y_{\Delta}(t)$ with modified coefficients given by (5.6) is also mean-square exponentially stable 160 with exponent $-\left(\gamma_{\Delta}^{\star} \wedge \frac{2}{\tau} \log \frac{1}{v}\right)$ for any $\Delta \in\left(0, \Delta^{\star}\right]$. Fig.1(b) plots the sample paths of tamed EM solutions $Y_{\Delta}(t)$ applied to NSDDE (6.8) for 200 simulations with step size $\Delta=0.001$ and $\alpha=1 / 4$. We see from Fig. $1(b)$ and Table 1 that the numerical solution is stable and $\gamma_{\Delta}^{\star}$ tends to $\gamma^{\star}$ as $\Delta$ goes to zero. Our experiments confirm the conclusion from Theorem 5.5 .

\section{Conclusion}

In this work, we mainly examine the strong convergence and stability of tamed EM scheme for NSDDEs, where the drift and diffusion coefficients may be allowed to grow superlinearly. By virtue of tamed technology, uniform boundedness of numerical solutions is obtained and then strong convergence results are established. The results show that the tamed EM approximation $Y(t)$ can arrive at an order one half of strong convergence. Meanwhile, it is proved that the tamed

170 EM solution has the property of reproduction of mean-square stability for the exact solution. Numerical experiments are provided to show the agreement with the theoretical results.

\section{Acknowledgments}

The authors would like to thank the referees for their very professional comments and helpful suggestions. This work is supported by the Natural Science Foundation of China (No.

175 71571001), the Royal Society (WM160014, Royal Society Wolfson Research Merit Award), the Royal Society and the Newton Fund (NA160317, Royal Society-Newton Advanced Fellowship), the EPSRC (EP/K503174/1). This work is entirely theoretical and the results can be reproduced using the methods described in this paper.

\section{References}

[1] M. Shen, W. Fei, X. Mao, Y. Liang, Stability of highly nonlinear neutral stochastic differential delay equations, Systems and Control Letters 115 (2018) 1-8.

[2] W. Mao, X. Mao, On the approximations of solutions to neutral SDEs with Markovian switching and jumps under non-Lipschitz conditions, Applied Mathematics and Computation 230 (2014) 104-119.

[3] X. Li, W. Cao, On mean-square stability of two-step Maruyama methods for nonlinear neutral stochastic delay differential equations, Applied Mathematics and Computation 261 (2015) 373-381.

[4] H. Mo, F. Deng, C. Zhang, Exponential stability of the split-step theta-method for neutral stochastic delay differential equations with jumps, Applied Mathematics and Computation 315 (2017) 85-95.

[5] Y. Ji, C. Yuan, Tamed EM scheme of neutral stochastic differential delay equations, Journal of Computational and Applied Mathematics 326 (2017) 337-357.

190 [6] X. Mao, The truncated Euler-Maruyama method for stochastic differential equations, Journal of Computational and Applied Mathematics 290 (2015) 370-384.

[7] G. Lan, F. Xia, Strong convergence rates of modified truncated EM method for stochastic differential equations, Journal of Computational and Applied Mathematics 334 (2018) 1-17.

[8] G. Lan, Asymptotic exponential stability of modified truncated EM method for neutral stochastic differential delay equations, Journal of Computational and Applied Mathematics 340 (2018) 334-341.

[9] S. Zhou, H. Jin, Numerical solution to highly nonlinear neutral-type stochastic differential equation, Applied Numerical Mathematics 140 (2019) 48-75.

[10] X. Zong, F. Wu, Exponential stability of the exact and numerical solutions for neutral stochastic delay differential equations, Applied Mathematical Modelling 40 (2016) 19-30. 
[11] L. Tan, Almost sure convergence rate of theta-EM scheme for neutral SDDEs, Journal of Computational and Applied Mathematics 342 (2018) 25-36.

[12] M. Milosevic, Highly nonlinear neutral stochastic differential equations with time-dependent delay and the EulerMaruyama method, Mathematical and Computer Modelling 54 (2011) 2235-2251.

[13] M. Milosevic, Almost sure exponential stability of solutions to highly nonlinear neutral stochastic differential equations with time-dependent delay and the Euler-Maruyama approximation, Mathematical and Computer Modelling 57 (2013) 887-899.

[14] L. Liu, Q. Zhu, Mean square stability of two classes of theta method for neutral stochastic differential delay equations, Journal of Computational and Applied Mathematics 305 (2016) 55-67.

[15] L. Tan, C. Yuan, Convergence rates of theta-method for NSDDEs under non-globally Lipschitz continuous coefficients, Bulletin of Mathematical Sciences 09 (2019) 1950006

[16] Z. Yan, A. Xiao, X. Tang, Strong convergence of the split-step theta method for neutral stochastic delay differential equations, Applied Numerical Mathematics 120 (2017) 215-232.

[17] Y. Xie, C. Zhang, Asymptotical boundedness and moment exponential stability for stochastic neutral differential equations with time-variable delay and Markovian switching, Applied Mathematics Letters 70 (2017) 46-51.

18] W. Zhang, Convergence rate of the truncated Euler-Maruyama method for neutral stochastic differential delay equations with Markovian switching, Journal of Computational Mathematics 38 (2020) 874-904.

[19] L. Tan, C. Yuan, Strong convergence of a tamed theta scheme for NSDDEs with one-sided Lipschitz drift, Applied Mathematics and Computation 338 (2018) 607-623.

[20] M. Hutzenthaler, A. Jentzen, P. E. Kloeden, Strong convergence of an explicit numerical method for SDEs with nonglobally Lipschitz continuous coefficients, The Annals of Applied Probability 22 (2012) 1611-1641.

[21] S. Sabanis, Euler approximations with varying coefficients: the case of superlinearly growing diffusion coefficients, The Annals of Applied Probability 26 (2016) 2083-2105.

[22] K. Dareiotis, C. Kumar, S. Sabanis, On tamed Euler approximations of SDEs driven by Lévy noise with applications to delay equations, SIAM Journal on Numerical Analysis 54 (2016) 1840-1872.

[23] C. Kumar, S. Sabanis, On Milstein approximations with varying coefficients: the case of super-linear diffusion coefficients, BIT Numerical Mathematics 59 (2019) 929-968.

[24] S. Sabanis, Y. Zhang, On explicit order 1.5 approximations with varying coefficients: The case of super-linear diffusion coefficients, Journal of Complexity 50 (2019) 84-115.

[25] M. V. Tretyakov, Z. Zhang, A fundamental mean-square convergence theorem for SDEs with locally Lipschitz coefficients and its applications, SIAM J.Numer.Anal 51 (2013) 3135-3162.

[26] Z. Zhang, H. Ma, Order-preserving strong schemes for SDEs with locally Lipschitz coefficients, Applied Numerical Mathematics 112 (2017) 1-16.

[27] W. Cao, J. Liang, Y. Liu, On strong convergence of explicit numerical methods for stochastic delay differential equations under non-global Lipschitz conditions, Journal of Computational and Applied Mathematics 382 (2021) 113079.

[28] Z. Chen, S. Gan, X. Wang, Mean-square approximations of Lévy noise driven SDEs with super-linearly growing diffusion and jump coefficients, Discrete and Continuous Dynamical Systems-B 24 (2019) 4513-4545.

[29] X. Mao, Convergence rates of the truncated Euler-Maruyama method for stochastic differential equations, Journal of Computational and Applied Mathematics 296 (2016) 362-375.

30] S. Deng, W. Fei, W. Liu, X. Mao, The truncated EM method for stochastic differential equations with Poisson jumps, Journal of Computational and Applied Mathematics 355 (2019) 232-257.

[31] H. Yang, F. Wu, P. E. Kloeden, X. Mao, The truncated Euler-Maruyama method for stochastic differential equations with Holder diffusion coefficients, Journal of Computational and Applied Mathematics 366 (2020) 112379

[32] W. Fei, L. Hu, X. Mao, D. Xia, Advances in the truncated Euler-Maruyama method for stochastic differential delay equations, Communications on Pure and Applied Analysis 19 (2020) 2081-2100.

[33] X. Li, X. Mao, G. Yin, Explicit numerical approximations for stochastic differential equations in finite and infinite horizons: truncation methods, convergence in $p$ th moment and stability, IMA Journal of Numerical Analysis 39 (2019) 847-892.

[34] Q. Guo, X. Mao, R. Yue, The truncated Euler-Maruyama method for stochastic differential delay equations, Numerical Algorithms 78 (2018) 599-624.

[35] Q. Guo, W. Liu, X. Mao, R. Yue, The partially truncated Euler-Maruyama method and its stability and boundedness, Appl. Numer. Math. 115 (2017) 235-251.

[36] N. Halidias, Semi-discrete approximations for stochastic differential equations and applications, International Journal of Computer Mathematics 89 (2012) 780-794

37] N. Halidias, I. S. Stamatiou, On the numerical solution of some non-linear stochastic differential equations using the semi-discrete method, Computational Methods in Applied Mathematics 16 (2016) 105-132.

[38] I. S. Stamatiou, An explicit positivity preserving numerical scheme for CIR/CEV type delay models with jump, Journal of Computational and Applied Mathematics 360 (2019) 78-98. 
[39] N. Halidias, I. S. Stamatiou, Convergence rates of the semi-discrete method for stochastic differential equations, Theory of Stochastic Processes 24 (2019) 89-100.

[40] X. Mao, Stochastic Differential Equations and Applications, Horwood, Chichester, 2nd edition, 2007. 